\title{
Surface operators, dual quivers and contours
}

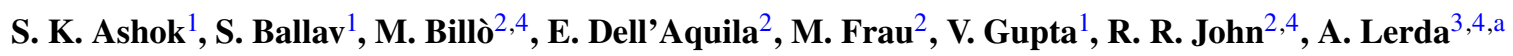 \\ ${ }^{1}$ Institute of Mathematical Sciences, Homi Bhabha National Institute (HBNI), IV Cross Road, C.I.T. Campus, Taramani, Chennai, \\ Tamil Nadu 600113, India \\ ${ }^{2}$ Dipartimento di Fisica, Università di Torino, Via P. Giuria 1, 10125 Torino, Italy \\ ${ }^{3}$ Dipartimento di Scienze e Innovazione Tecnologica, Università del Piemonte Orientale, Viale T. Michel 11, 15121 Alessandria, Italy \\ ${ }^{4}$ Arnold-Regge Center and I.N.F.N.-sezione di Torino, Via P. Giuria 1, 10125 Torino, Italy
}

Received: 6 February 2019 / Accepted: 17 March 2019 / Published online: 26 March 2019

(C) The Author(s) 2019

\begin{abstract}
We study half-BPS surface operators in four dimensional $\mathcal{N}=2 \mathrm{SU}(N)$ gauge theories, and analyze their low-energy effective action on the four dimensional Coulomb branch using equivariant localization. We also study surface operators as coupled $2 \mathrm{~d} / 4 \mathrm{~d}$ quiver gauge theories with an $\mathrm{SU}(N)$ flavour symmetry. In this description, the same surface operator can be described by different quivers that are related to each other by two dimensional Seiberg duality. We argue that these dual quivers correspond, on the localization side, to distinct integration contours that can be determined by the Fayet-Iliopoulos parameters of the two dimensional gauge nodes. We verify the proposal by mapping the solutions of the twisted chiral ring equations of the $2 \mathrm{~d} / 4 \mathrm{~d}$ quivers onto individual residues of the localization integrand.
\end{abstract}

\section{Introduction}

Surface operators in $4 \mathrm{~d}$ gauge theories are natural two dimensional generalizations of Wilson and 't Hooft loops which can provide valuable information about the phase structure of the gauge theories [1]. In this paper we study the lowenergy effective action of surface operators in pure $\mathcal{N}=2$ $4 \mathrm{~d}$ gauge theories from two distinct points of view, namely as monodromy defects $[2,3]$ and as coupled $2 \mathrm{~d} / 4 \mathrm{~d}$ quiver gauge theories $[4,5]$. In the first approach, one specifies how the $4 \mathrm{~d}$ gauge fields are affected by the presence of the surface operator by imposing suitable boundary conditions in the path-integral. In this framework the non-perturbative effects are described in terms of ramified instantons [2] whose partition function can be computed using equivariant localization methods [5-10]. From the ramified instanton partition function one can extract two holomorphic functions [11,12]: one is the prepotential $\mathcal{F}$ that governs the low-energy effective

\footnotetext{
a e-mail: lerda@to.infn.it
}

action of the $4 \mathrm{~d} \mathcal{N}=2$ gauge theory on the Coulomb branch; the other is the twisted chiral superpotential $\mathcal{W}$ that describes the $2 \mathrm{~d}$ dynamics on the defect.

In the second description of the surface operators, one considers coupled $2 \mathrm{~d} / 4 \mathrm{~d}$ theories that are $(2,2)$ supersymmetric sigma models with an ultraviolet description as a gauged linear sigma model (GLSM). The low-energy dynamics of such a GLSM is completely determined by a twisted chiral superpotential $\mathcal{W}(\sigma)$ that depends on the twisted chiral superfields $\sigma$ containing the $2 \mathrm{~d}$ vector fields [13]. By giving a vacuum expectation value (vev) to the adjoint scalar of the $4 \mathrm{~d} \mathcal{N}=2$ gauge theory, one introduces twisted masses in the $2 \mathrm{~d}$ quiver theory $[14,15]$. At a generic point on the $4 \mathrm{~d}$ Coulomb branch, the $2 \mathrm{~d}$ theory is therefore massive in the infrared and the $2 \mathrm{~d} / 4 \mathrm{~d}$ coupling mechanism is determined via the resolvent of the $4 \mathrm{~d}$ gauge theory [5]. The resulting massive vacua of the GLSM are solutions to the twisted chiral ring equations, which are obtained by extremizing $\mathcal{W}(\sigma)$ with respect to the twisted chiral superfields.

The main goal of this work is to clarify the precise relationship between the above two descriptions of the surface operators. In our previous works $[9,10]$ the first steps in this direction were already taken by showing that there is a precise correspondence between the massive vacua of the $2 \mathrm{~d} / 4 \mathrm{~d}$ gauge theory and the monodromy defects in the $\mathcal{N}=2$ gauge theory. In fact, the effective twisted chiral superpotential of the $2 \mathrm{~d} / 4 \mathrm{~d}$ quiver gauge theory evaluated in a given massive vacuum exactly coincides with the one computed from the $4 \mathrm{~d}$ ramified instanton partition function $[9,10]$. This equality was shown in a specific class of models that are described by oriented quiver diagrams. Recently, this result has been proven in full generality in $[16,17]$.

An important feature of the $(2,2)$ quiver theories that was not fully discussed in our previous papers is Seiberg duality $[18,19]$. This is an infrared equivalence between two gauge theories that have different ultraviolet realizations. In 
this work we fill this gap and consider all possible quivers obtained from the oriented ones by applying $2 \mathrm{~d}$ Seiberg duality. While all such quivers have different gauge groups and matter content, once the $4 \mathrm{~d}$ Coulomb vev's are turned on, it is possible to find a one-to-one map between their massive vacua. Therefore it becomes clear that they must describe the same surface operator from the point of view of the $4 \mathrm{~d}$ gauge theory; indeed, the different twisted chiral superpotentials, evaluated in the respective vacua, all give the same result. This equality of superpotentials gives a strong hint that the choice of a Seiberg duality frame might have an interpretation as distinct contours of integration on the localization side: the equality of the superpotentials would then be a simple consequence of multi-dimensional residue theorems.

In this work we show that this expectation is correct and provide a detailed map between a given quiver realization of the surface operator and a particular choice of contour in the localization integrals. This contour prescription can be conveniently encoded in a Jeffrey-Kirwan (JK) reference vector [20], whose coefficients turn out to be related to the FayetIliopoulos (FI) parameters of the $2 \mathrm{~d} / 4 \mathrm{~d}$ quiver. While the twisted superpotentials are equal irrespective of the choice of contour, the map relates the individual residues on the localization side to the individual terms in the solutions to the twisted chiral ring equations, thereby allowing us to identify in an unambiguous way which quiver arises from a given contour prescription and vice-versa.

This paper is organized as follows. In Sect. 2 we review and extend our earlier work [8-10], and in particular we show how to map the oriented quiver to a particular contour by studying the solution of the chiral ring equations and the precise correspondence to the residues of the localization integrand. In Sect. 3 we discuss the basics of $2 \mathrm{~d}$ Seiberg duality and how it acts on the quiver theories we consider. In Sect. 4 we apply the duality moves to the oriented quiver of interest and show in detail (for the 4-node quiver), how it is possible to map each quiver to a particular integration contour on the localization side without explicitly solving the chiral ring equations. We also discuss how this integration contour can be specified in terms of a JK reference vector. In Sect. 5 we give a simple solution for the $\mathrm{JK}$ vector associated to a generic linear $2 \mathrm{~d} / 4 \mathrm{~d}$ quiver with arbitrary number of nodes. Finally, we summarize our main results in Sect. 6 and collect the more technical material in the appendices.

\section{Review of earlier work}

To set the stage for the discussion in the next sections and also to introduce our notation, we briefly review the results obtained in our earlier work [10] where we studied surface operators both as monodromy defects in $4 \mathrm{~d}$ and as coupled $2 \mathrm{~d} / 4 \mathrm{~d}$ gauge theories.
2.1 Surface operators as monodromy defects

As a monodromy defect, a surface operator in a $4 \mathrm{~d} \operatorname{SU}(N)$ theory is specified by a partition of $N$, denoted by $\boldsymbol{n}=$ $\left(n_{1}, n_{2}, \ldots n_{M}\right)$, which corresponds to the breaking of the gauge group to a Levi subgroup

$\mathbb{L}=\mathrm{S}\left[\mathrm{U}\left(n_{1}\right) \times \mathrm{U}\left(n_{2}\right) \times \cdots \mathrm{U}\left(n_{M}\right)\right]$

at the location of the defect [2,3]. This also gives a natural partitioning of the classical Coulomb vev's of the adjoint scalar $\Phi$ of the $\mathcal{N}=2 \mathrm{SU}(N)$ theory as follows:

$\langle\Phi\rangle=\left\{a_{1}, \ldots, a_{r_{1}}|\ldots| a_{r_{I-1}+1}, \ldots a_{r_{I}}|\ldots| a_{r_{M-1}+1}, \ldots, a_{N}\right\}$.

Here we have defined the integers $r_{I}$ according to

$r_{I}=\sum_{J=1}^{I} n_{J}$

so that the $I$ th partition in (2) is of length $n_{I}$. Introducing the following set of numbers with cardinality $n_{I}$ :

$\mathcal{N}_{I} \equiv\left\{r_{I-1}+1, r_{I-1}+2, \ldots, r_{I}\right\}$

we define the $n_{I} \times n_{I}$ block-diagonal matrices $\mathcal{A}_{I}$ according to

$\mathcal{A}_{I} \equiv \operatorname{diag}\left(a_{s \in \mathcal{N}_{I}}\right)=\left(\begin{array}{cccc}a_{r_{I-1}+1} & 0 & 0 & \ldots \\ 0 & \ddots & 0 & \ldots \\ \vdots & \vdots & \ddots & \\ 0 & 0 & \ldots & a_{r_{I}}\end{array}\right)$.

With these conventions, the splitting in (2) can be written as

$\langle\Phi\rangle=\mathcal{A}_{1} \oplus \mathcal{A}_{2} \oplus \ldots \oplus \mathcal{A}_{M}$.

The instanton partition function in the presence of such a surface operator, also known as the ramified instanton partition function, takes the following form $[6,10]$ :

$Z_{\text {inst }}[\boldsymbol{n}]=\sum_{\left\{d_{I}\right\}} Z_{\left\{d_{I}\right\}}[\boldsymbol{n}]$

with

$Z_{\left\{d_{I}\right\}}[\boldsymbol{n}]=\prod_{I=1}^{M}\left[\frac{\left(-q_{I}\right)^{d_{I}}}{d_{I} !} \int \prod_{\sigma=1}^{d_{I}} \frac{d \chi_{I, \sigma}}{2 \pi \mathrm{i}}\right] z_{\left\{d_{I}\right\}}$

where

$$
\begin{aligned}
z_{\left\{d_{I}\right\}}= & \prod_{I=1}^{M} \prod_{\sigma, \tau=1}^{d_{I}} \frac{\left(\chi_{I, \sigma}-\chi_{I, \tau}+\delta_{\sigma, \tau}\right)}{\left(\chi_{I, \sigma}-\chi_{I, \tau}+\epsilon_{1}\right)} \\
& \times \prod_{I=1}^{M} \prod_{\sigma=1}^{d_{I}} \prod_{\rho=1}^{d_{I+1}} \frac{\left(\chi_{I, \sigma}-\chi_{I+1, \rho}+\epsilon_{1}+\hat{\epsilon}_{2}\right)}{\left(\chi_{I, \sigma}-\chi_{I+1, \rho}+\hat{\epsilon}_{2}\right)}
\end{aligned}
$$




$$
\begin{aligned}
& \times \prod_{I=1}^{M} \prod_{\sigma=1}^{d_{I}} \frac{1}{\prod_{s \in \mathcal{N}_{I}}\left(a_{s}-\chi_{I, \sigma}+\frac{1}{2}\left(\epsilon_{1}+\hat{\epsilon}_{2}\right)\right)} \\
& \times \prod_{I=1}^{M} \prod_{\sigma=1}^{d_{I}} \frac{1}{\prod_{t \in \mathcal{N}_{I+1}}\left(\chi_{I, \sigma}-a_{t}+\frac{1}{2}\left(\epsilon_{1}+\hat{\epsilon}_{2}\right)\right)} .
\end{aligned}
$$

Here, the $M$ positive integers $d_{I}$ count the numbers of ramified instantons in the various sectors, the variables $q_{I}$ are the ramified instanton weights, and the parameters $\epsilon_{1}$ and $\hat{\epsilon}_{2}=\epsilon_{2} / M$ specify the $\Omega$-background [21,22] which is introduced to localize the integrals over the instanton moduli space. $^{1}$

There is one more ingredient that is needed to calculate the partition function (8), namely the contour of integration for the $\chi_{I}$ variables. A convenient way to specify it and to select which poles of the integrand contribute and which do not, is to treat the Coulomb vev's $a$ as real variables and assign an imaginary part to the $\Omega$-deformation parameters according to

$0<\operatorname{Im}\left(\hat{\epsilon}_{2}\right) \ll \operatorname{Im}\left(\epsilon_{1}\right) \ll 1$.

Then, the contour is specified by integrating $\chi_{I, \sigma}$ in the upper or lower half-plane and by choosing a definite order in the successive integrations. Equivalently, as we will see in the following sections, the contour of integration can be selected by specifying a Jeffrey-Kirwan reference vector [20].

In the limit $\epsilon_{1}, \hat{\epsilon}_{2} \rightarrow 0$, the low-energy effective action of the gauge theory with the $2 \mathrm{~d}$ defect is specified by two holomorphic functions: the prepotential $\mathcal{F}$ and the twisted chiral superpotential $\mathcal{W}$. Each of these functions can be written as a sum of the classical, the one-loop, and the instanton contributions. The latter can be extracted from the ramified instanton partition function as follows $[11,12]$ :

$\log \left(1+Z_{\text {inst }}\right)=-\frac{\mathcal{F}_{\text {inst }}}{\epsilon_{1} \hat{\epsilon}_{2}}+\frac{\mathcal{W}_{\text {inst }}}{\epsilon_{1}}+\ldots$

where the ellipses refer to regular terms. In Appendix A we list the one-instanton contribution to $\mathcal{W}_{\text {inst }}$ calculated for various choices of contours in the case $M=4$. As we will show in the following, the different contour prescriptions can be given a precise meaning by associating them to specific $2 \mathrm{~d} / 4 \mathrm{~d}$ quiver gauge theories.

\subsection{Surface operators as coupled $2 \mathrm{~d} / 4 \mathrm{~d}$ quivers}

The prepotential $\mathcal{F}$ governs the 4 d gauge theory dynamics at a generic point on the Coulomb branch. The twisted chiral superpotential $\mathcal{W}$, instead, is best understood as the lowenergy effective description of a $2 \mathrm{~d}$ non-linear sigma model.

1 The rescaling by a factor of $M$ in $\epsilon_{2}$ is due to a $\mathbb{Z}_{M}$-orbifold projection that has to be performed in the ramified instanton case [6]. Furthermore, in (9) the sub-index $I$ is always understood modulo $M$.

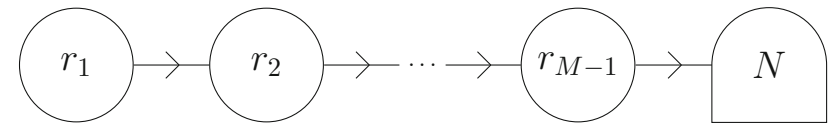

Fig. 1 The quiver which describes the generic surface operator in pure $\mathrm{SU}(N)$ gauge theory

For a surface operator with a Levi subgroup $\mathbb{L}$ in a $4 d$ theory with a gauge group $G$, the relevant sigma model is defined on the target space $G / \mathbb{L}[2,3]$. Such a space is, in general, a flag variety which can be realized as the low-energy limit of a GLSM [13,15], whose gauge and matter content can be summarized in the quiver diagram of Fig. 1.

Each circular node represents a $2 \mathrm{~d}$ gauge group $\mathrm{U}\left(r_{I}\right)$ where the ranks $r_{I}$ are as in (3), whereas the last node on the right hand side represents the $4 \mathrm{~d}$ gauge group $\mathrm{SU}(N)$ which acts as a flavour symmetry group for the $(M-1)^{\text {th }} 2 \mathrm{~d}$ node. The arrows correspond to matter multiplets which are rendered massive by non-zero v.e.v's of the twisted scalars $\sigma^{(I)}$ of the $I^{\text {th }}$ node and of the $4 \mathrm{~d}$ adjoint scalar $\Phi$. The orientation of the arrows specifies whether the matter is in the fundamental (out-going) or in the anti-fundamental (ingoing) representation.

The effective action for the twisted chiral multiplets is obtained by integrating out the massive matter multiplets and, thanks to supersymmetry, can be encoded in the effective twisted chiral superpotential. For the quiver of Fig. 1, this is given by:

$$
\begin{aligned}
\mathcal{W}= & 2 \pi \mathrm{i} \sum_{I=1}^{M-1} \sum_{s=1}^{r_{I}} \tau_{I} \sigma_{s}^{(I)}-\sum_{I=1}^{M-2} \sum_{s=1}^{r_{I}} \sum_{t=1}^{r_{I+1}} \varpi\left(\sigma_{s}^{(I)}-\sigma_{t}^{(I+1)}\right) \\
& -\sum_{s=1}^{r_{M-1}}\left\langle\operatorname{Tr} \varpi\left(\sigma_{s}^{(M-1)}-\Phi\right)\right\rangle
\end{aligned}
$$

where

$\varpi(x)=x\left(\log \frac{x}{\mu}-1\right)$,

$\mu$ is the UV cut-off scale, and $\tau_{I}$ is the complexified FI parameter of the $I$ th node at the scale $\mu$, namely

$\tau_{I}=\frac{\theta_{I}}{2 \pi}+\mathrm{i} \zeta_{I}$

with $\theta_{I}$ and $\zeta_{I}$ being, respectively, the $\theta$-parameter and the real FI parameter of the $I$ th gauge node. Finally, the angular brackets in the last term of (12) correspond to a chiral correlator in the $4 \mathrm{~d} \mathrm{SU}(N)$ theory. This correlator implies that the coupling between the $2 \mathrm{~d}$ and $4 \mathrm{~d}$ theory is via the resolvent of the $\mathrm{SU}(N)$ gauge theory [5], which in turn depends on the $4 d$ dynamically generated scale $\Lambda_{4 \mathrm{~d}}$. 
The running of the FI parameters leads to introducing $2 \mathrm{~d}$ low-energy scales $\Lambda_{I}$ at each node by the relation

$\Lambda_{I}^{b_{I}}=\mathrm{e}^{2 \pi \mathrm{i} \tau_{I}} \mu^{b_{I}}$

where $b_{I}$ is the corresponding $\beta$-function coefficient, which in this case is

$b_{I}=n_{I}+n_{I+1}$.

Of course, we can rewrite (15) as

$\left|\frac{\Lambda_{I}}{\mu}\right|=\mathrm{e}^{-2 \pi \frac{\zeta_{I}}{b_{I}}}$

which implies that

$\frac{\zeta_{I}}{b_{I}}>0$

Since for the quiver represented in Fig. 1, all $b_{I}$ are positive (see (16)), we deduce that

$\zeta_{I}>0$.

Once the $4 \mathrm{~d}$ Coulomb vev's are given, the $2 \mathrm{~d}$ Coulomb branch is completely lifted except for a finite number of discrete vacua. These are found by extremizing the twisted chiral superpotential $\mathcal{W}$, i.e. they are solutions of the twisted chiral ring equations [23,24]

$\exp \left(\frac{\partial \mathcal{W}}{\partial \sigma_{s}^{(I)}}\right)=1$

In order to make contact with the partition of the vev's in (2), we solve (20) about the following classical vacuum:

$\sigma_{\mathrm{cl}}^{(I)}=\mathcal{A}_{1} \oplus \mathcal{A}_{2} \oplus \cdots \oplus \mathcal{A}_{I}$.

Once the solutions to the twisted chiral ring equations are obtained (order by order in the low-energy scales of the $2 \mathrm{~d} / 4 \mathrm{~d}$ theories), we evaluate the effective twisted chiral superpotential $\mathcal{W}$ on this particular solution, and verify that the nonperturbative contributions exactly coincide with the $\mathcal{W}_{\text {inst }}$ calculated using localization. In essence, this match provides a one-to-one map between $\frac{1}{2}$-BPS defects in the $4 \mathrm{~d}$ gauge theory and massive vacua in the coupled $2 \mathrm{~d} / 4 \mathrm{~d}$ gauge theory.

\subsection{A contour from the twisted chiral ring}

We now consider in detail the case $M=4$ corresponding to the quiver in Fig. 2. This is the simplest example that is general enough to contain all relevant features of a generic linear quiver, and thus it serves as a prototypical case.

The twisted chiral ring Eq. (20) can be compactly written in terms of a characteristic gauge polynomial for each $\mathrm{U}\left(r_{I}\right)$ node, given by

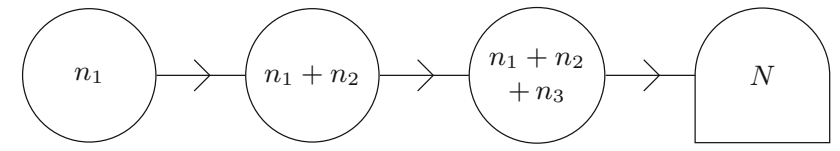

Fig. 2 The 4-node linear quiver that corresponds to the partition $\left[n_{1}, n_{2}, n_{3}, n_{4}\right]$

$\mathcal{Q}_{I}(z)=\prod_{s=1}^{r_{I}}\left(z-\sigma_{s}^{(I)}\right)$,

and the characteristic polynomial of the $4 \mathrm{~d} \operatorname{SU}(N)$ node, namely

$\mathcal{P}_{N}(z)=z^{N}+\sum_{i=2}^{N}(-1)^{k} u_{k} z^{N-k}$.

Here $u_{k}$ are the gauge invariant coordinates on the moduli space, which can be calculated at weak coupling using localization methods [25-29]. In terms of these polynomials, the twisted chiral equations (20) become [10]

$$
\begin{aligned}
\mathcal{Q}_{2}\left(\sigma_{s}^{(1)}\right)= & \Lambda_{1}^{n_{1}+n_{2}}, \\
\mathcal{Q}_{3}\left(\sigma_{t}^{(2)}\right)= & (-1)^{n_{1}} \Lambda_{2}^{n_{2}+n_{3}} \mathcal{Q}_{1}\left(\sigma_{t}^{(2)}\right), \\
\mathcal{P}_{N}\left(\sigma_{u}^{(3)}\right)= & (-1)^{n_{1}+n_{2}}\left(\Lambda_{3}^{n_{3}+n_{4}} \mathcal{Q}_{2}\left(\sigma_{u}^{(3)}\right)\right. \\
& \left.+\frac{\Lambda_{4 \mathrm{~d}}^{2 N}}{\Lambda_{3}^{n_{3}+n_{4}} \mathcal{Q}_{2}\left(\sigma_{u}^{(3)}\right)}\right),
\end{aligned}
$$

for $s \in \mathcal{N}_{1}, t \in \mathcal{N}_{1} \cup \mathcal{N}_{2}$, and $u \in \mathcal{N}_{1} \cup \mathcal{N}_{2} \cup \mathcal{N}_{3}$, respectively. We look for solutions of these equations that are of the form

$\sigma_{\star}^{(I)}=\sigma_{\mathrm{cl}}^{(I)}+\delta \sigma^{(I)}$

where the classical part is as in (21) for $I=1,2,3$. A detailed derivation of the solution at the one-instanton level is presented in Appendix B. Here we merely write the expressions for the non-vanishing first-order corrections, that are

$$
\begin{aligned}
\delta \sigma_{s}^{(1)}= & \frac{\Lambda_{1}^{n_{1}+n_{2}}}{\prod_{r \in \widehat{\mathcal{N}}_{1} \cup \mathcal{N}_{2}}\left(a_{s}-a_{r}\right)} \\
& +\frac{(-1)^{n_{2}} \Lambda_{4 \mathrm{~d}}^{2 N}}{\Lambda_{1}^{n_{1}+n_{2}} \Lambda_{2}^{n_{2}+n_{3}} \Lambda_{3}^{n_{3}+n_{4}} \prod_{r \in \mathcal{N}_{4} \cup \widehat{\mathcal{N}}_{1}}\left(a_{s}-a_{r}\right)}, \\
\delta \sigma_{s}^{(2)}= & \delta \sigma_{s}^{(3)}=\frac{(-1)^{n_{2}} \Lambda_{4 \mathrm{~d}}^{2 N}}{\Lambda_{1}^{n_{1}+n_{2}} \Lambda_{2}^{n_{2}+n_{3}} \Lambda_{3}^{n_{3}+n_{4}} \prod_{r \in \mathcal{N}_{4} \cup \widehat{\mathcal{N}}_{1}}\left(a_{s}-a_{r}\right)}
\end{aligned}
$$

for $s \in \mathcal{N}_{1}$,

$\delta \sigma_{t}^{(2)}=\frac{(-1)^{n_{1}} \Lambda_{2}^{n_{2}+n_{3}}}{\prod_{r \in \widehat{\mathcal{N}}_{2} \cup \mathcal{N}_{3}}\left(a_{t}-a_{r}\right)}$ 
for $t \in \mathcal{N}_{2}$, and

$\delta \sigma_{u}^{(3)}=\frac{(-1)^{n_{1}+n_{2}} \Lambda_{3}^{n_{3}+n_{4}}}{\prod_{r \in \widehat{\mathcal{N}}_{3} \cup \mathcal{N}_{4}}\left(a_{u}-a_{r}\right)}$

for $u \in \mathcal{N}_{3}$. In these formulas, the symbol $\widehat{\mathcal{N}}_{I}$ means that one has to omit from the set $\mathcal{N}_{I}$ the indices that would yield a vanishing denominator.

In [10] it was shown that

$\operatorname{Tr} \sigma_{\star}^{(I)}=\left.\frac{1}{b_{I}} \Lambda_{I} \frac{\partial \mathcal{W}}{\partial \Lambda_{I}}\right|_{\sigma_{\star}}$.

Integrating in this relation, one can obtain the twisted superpotential in the chosen vacuum, which in the one-instanton approximation is

$$
\begin{aligned}
\left.\mathcal{W}\right|_{\sigma_{\star}}= & \sum_{s \in \mathcal{N}_{1}} \frac{\Lambda_{1}^{n_{1}+n_{2}}}{\prod_{r \in \widehat{\mathcal{N}}_{1} \cup \mathcal{N}_{2}}\left(a_{s}-a_{r}\right)} \\
& +\sum_{t \in \mathcal{N}_{2}} \frac{(-1)^{n_{1}} \Lambda_{2}^{n_{2}+n_{3}}}{\prod_{r \in \widehat{\mathcal{N}}_{2} \cup \mathcal{N}_{3}}\left(a_{t}-a_{r}\right)} \\
& +\sum_{u \in \mathcal{N}_{3}} \frac{(-1)^{n_{1}+n_{2}} \Lambda_{3}^{n_{3}+n_{4}}}{\prod_{r \in \widehat{\mathcal{N}}_{3} \cup \mathcal{N}_{4}}\left(a_{u}-a_{r}\right)} \\
& +\sum_{s \in \mathcal{N}_{1}} \frac{(-1)^{n_{2}+1} \Lambda_{4 \mathrm{~d}}^{2 N}}{\Lambda_{1}^{n_{1}+n_{2}} \Lambda_{2}^{n_{2}+n_{3}} \Lambda_{3}^{n_{3}+n_{4}} \prod_{r \in \mathcal{N}_{4} \cup \widehat{\mathcal{N}}_{1}}\left(a_{s}-a_{r}\right)} .
\end{aligned}
$$

We now compare this expression with the result of the localization analysis at the one ramified instanton level. From (8) and (9), specified to the partition $\left[n_{1}, \ldots, n_{4}\right]$, we find

$$
\begin{aligned}
Z_{1-\mathrm{inst}}= & -\sum_{I=1}^{4} q_{I} \int \frac{d \chi_{I}}{2 \pi \mathrm{i}} \frac{1}{\epsilon_{1}} \prod_{s \in \mathcal{N}_{I}} \frac{1}{\left(a_{s}-\chi_{I}+\frac{1}{2}\left(\epsilon_{1}+\hat{\epsilon}_{2}\right)\right)} \\
& \times \prod_{t \in \mathcal{N}_{I+1}} \frac{1}{\left(\chi_{I}-a_{t}+\frac{1}{2}\left(\epsilon_{1}+\hat{\epsilon}_{2}\right)\right)}
\end{aligned}
$$

In view of the prescription (10), it is clear that the number of poles that contribute to a given $\chi_{I}$-integral depends upon whether we close the contour in the upper or lower halfplanes. Closing the contour in the upper half-plane leads to $n_{I}$ poles that contribute, while closing the contour in the lower half-plane leads to $n_{I+1}$ poles that contribute. Furthermore, the mass dimensions of each $q_{I}$ is fixed to be $n_{I}+n_{I+1}$, since the partition function itself is dimensionless. These two facts immediately help us in relating the localization results with the chiral ring analysis. ${ }^{2}$ Indeed, the dimensional argument

${ }^{2}$ In a purely $2 d$ context, a relation between the solution of chiral ring equations for certain quiver theories and contour integrals has been noticed in [30]. allows us to express the ramified instanton counting parameters in terms of the $2 \mathrm{~d}$ effective scales as follows $[10]^{3}$ :

$$
\begin{aligned}
& q_{1}=(-1)^{n_{1}} \Lambda_{1}^{n_{1}+n_{2}}, \\
& q_{2}=(-1)^{n_{1}+n_{2}} \Lambda_{2}^{n_{2}+n_{3}}, \\
& q_{3}=(-1)^{n_{1}+n_{2}+n_{3}} \Lambda_{3}^{n_{3}+n_{4}}, \\
& q_{4}=\frac{(-1)^{n_{2}+n_{4}} \Lambda_{4 \mathrm{~d}}^{2 N}}{\Lambda_{1}^{n_{1}+n_{2}} \Lambda_{2}^{n_{2}+n_{3}} \Lambda_{3}^{n_{3}+n_{4}}} .
\end{aligned}
$$

Using (15), the first three $q_{I}$ can also be written in terms of the bare complexified FI parameters $\tau_{I}$ of the three $2 \mathrm{~d}$ nodes as

$$
\begin{aligned}
& q_{1}=\mathrm{e}^{2 \pi \mathrm{i} \tau_{1}}(-1)^{n_{1}} \mu^{n_{1}+n_{2}}, \\
& q_{2}=\mathrm{e}^{2 \pi \mathrm{i} \tau_{2}}(-1)^{n_{1}+n_{2}} \mu^{n_{2}+n_{3}}, \\
& q_{3}=\mathrm{e}^{2 \pi \mathrm{i} \tau_{3}}(-1)^{n_{1}+n_{2}+n_{3}} \mu^{n_{3}+n_{4}} .
\end{aligned}
$$

Once the identification (32) is made, we can match the number and the structure of the terms that appear in (30) by closing the contours for $\chi_{1}, \chi_{2}$ and $\chi_{3}$ in the upper halfplane, and the contour of $\chi_{4}$ in the lower half-plane. We denote this choice of contours as $\left(\left.\chi_{1}\right|_{+},\left.\chi_{2}\right|_{+},\left.\chi_{3}\right|_{+},\left.\chi_{4}\right|_{-}\right)$. Indeed, computing the corresponding residues and extracting the one-instanton twisted superpotential from (11) and (31), we find

$$
\begin{aligned}
\mathcal{W}_{1-\text { inst }}= & \sum_{s \in \mathcal{N}_{1}} \frac{(-1)^{n_{1}} q_{1}}{\prod_{r \in \widehat{\mathcal{N}}_{1} \cup \mathcal{N}_{2}}\left(a_{s}-a_{r}\right)} \\
& +\sum_{t \in \mathcal{N}_{2}} \frac{(-1)^{n_{2}} q_{2}}{\prod_{r \in \widehat{\mathcal{N}}_{2} \cup \mathcal{N}_{3}}\left(a_{t}-a_{r}\right)} \\
& +\sum_{u \in \mathcal{N}_{3}} \frac{(-1)^{n_{3}} q_{3}}{\prod_{r \in \widehat{\mathcal{N}}_{3} \cup \mathcal{N}_{4}}\left(a_{u}-a_{r}\right)} \\
& +\sum_{s \in \mathcal{N}_{1}} \frac{(-1)^{n_{4}+1} q_{4}}{\prod_{r \in \mathcal{N}_{4} \cup \widehat{\mathcal{N}}_{1}}\left(a_{s}-a_{r}\right)}
\end{aligned}
$$

which, term by term, exactly matches the superpotential (30) obtained by solving the twisted chiral ring equations.

\section{2d Seiberg duality}

The notion of Seiberg duality in 4d gauge theories [18] can be generalized to two dimensions (see for example [19]). Thus, by applying $2 \mathrm{~d}$ Seiberg duality it is possible to obtain distinct quiver theories in the UV that have the same IR behaviour.

Let us first consider the simplest case, shown in Fig. 3.

This is a $2 \mathrm{~d} \mathrm{U}(r)$ gauge theory with $N_{F}$ fundamental flavours and $N_{A}$ anti-fundamental flavours. For definiteness we take $N_{F}>N_{A}$, and call this system "theory A". Its classical twisted superpotential is simply

\footnotetext{
3 The signs have been chosen to match the two superpotentials exactly.
} 


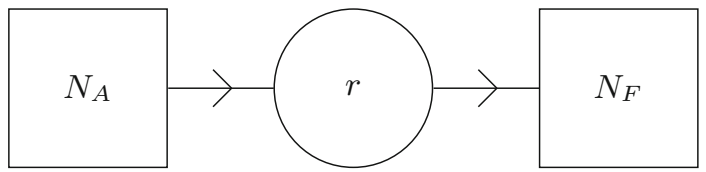

Fig. 3 A single $2 \mathrm{~d}$ gauge node of rank $r$ with $N_{F}$ fundamental and $N_{A}$ anti-fundamental flavours attached to it

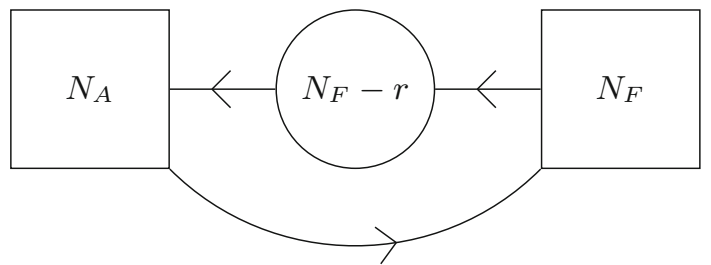

Fig. 4 The theory obtained after a $2 d$ Seiberg duality on the gauge node in Fig. 3

$\mathcal{W}_{\mathrm{cl}}^{\mathrm{A}}=2 \pi \mathrm{i} \tau \operatorname{Tr} \sigma$

We now perform a Seiberg duality, and obtain "theory B", which is described by the quiver in Fig. 4.

Under the duality, the rank of the gauge group changes as

$r \longrightarrow r^{\prime}=\max \left(N_{F}, N_{A}\right)-r=N_{F}-r$,

and the roles of the fundamental and anti-fundamental flavours are exchanged as denoted by the reversal of the arrows. The classical twisted superpotential for "theory B" is ${ }^{4}$

$\mathcal{W}_{\mathrm{cl}}^{\mathrm{B}}=-2 \pi \mathrm{i} \tau \operatorname{Tr} \sigma^{\prime}+2 \pi \mathrm{i} \tau \sum_{f=1}^{N_{F}} m_{f}$

where $\sigma^{\prime}$ denotes the twisted chiral superfield in the vector multiplet of the dualized node and $m_{f}$ are the twisted masses that completely break the flavour symmetry to its Cartan subgroup.

We now apply this basic duality rule to the quiver theories that describe surface operators. Since for a given $2 \mathrm{~d}$ node the flavour symmetry is realized by the adjacent nodes, we can encounter three kinds of configurations. The first one is when we dualize a gauge node with both fundamental and anti-fundamental fields in an oriented sequence, as shown in Fig. 5.

Before the duality, the classical superpotential for the three relevant nodes is

$$
\begin{aligned}
\mathcal{W}_{\mathrm{cl}}^{\mathrm{A}}= & \cdots+2 \pi \mathrm{i} \tau_{1} \operatorname{Tr} \sigma^{(1)}+2 \pi \mathrm{i} \tau \operatorname{Tr} \sigma \\
& +2 \pi \mathrm{i} \tau_{2} \operatorname{Tr} \sigma^{(2)}+\cdots,
\end{aligned}
$$

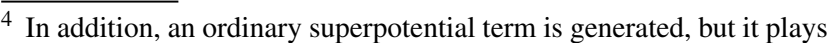
no role in our discussion.
}

(A)

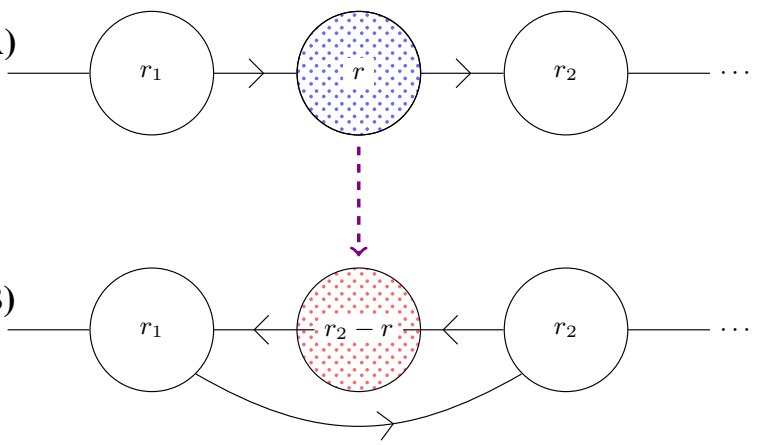

Fig. 52 d Seiberg duality on a node with both fundamental and antifundamental matter with $r_{2}>r_{1}$. The rank of the dualized node is $\max \left(r_{1}, r_{2}\right)-r=r_{2}-r$. The blue and red colours indicate the node before and after the duality

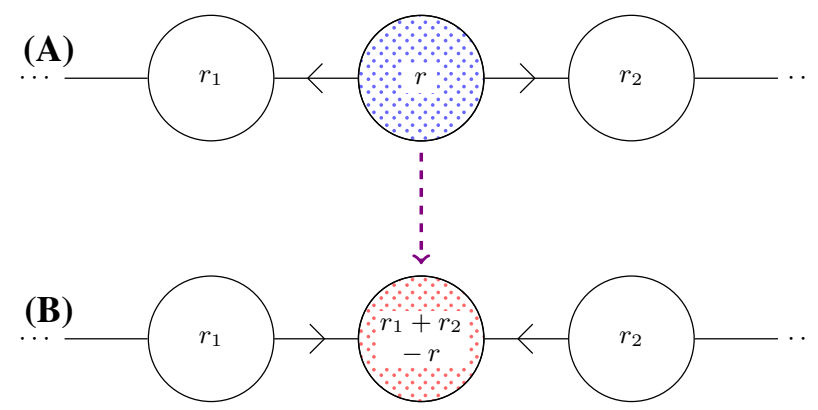

Fig. 6 2d Seiberg duality on a node with only chiral fundamental matter realized by adjacent $2 \mathrm{~d}$ gauge nodes. In this case there are no mesonic fields introduced in this case

while, after duality, it becomes

$$
\begin{aligned}
\mathcal{W}_{\mathrm{cl}}^{\mathrm{B}}= & \cdots+2 \pi \mathrm{i} \tau_{1} \operatorname{Tr} \sigma^{(1)}-2 \pi \mathrm{i} \tau \operatorname{Tr} \sigma^{\prime} \\
& +2 \pi \mathrm{i}\left(\tau_{2}+\tau\right) \operatorname{Tr} \sigma^{(2)}+\cdots .
\end{aligned}
$$

Here we have taken into account the fact that the role of the twisted masses for the dualized node is played by the $\sigma$ variables of the $r_{2}$ node. This explains why the FI parameter $\tau_{2}$ is shifted by $\tau$.

The second possibility is when we dualize a node with only fundamental matter, as shown in Fig. 6. In this case the classical superpotential before the duality is still given by (38), but after the duality it becomes

$$
\begin{aligned}
\mathcal{W}_{\mathrm{cl}}^{\mathrm{B}}= & \cdots+2 \pi \mathrm{i}\left(\tau_{1}+\tau\right) \operatorname{Tr} \sigma^{(1)}-2 \pi \mathrm{i} \tau \operatorname{Tr} \sigma^{\prime} \\
& +2 \pi \mathrm{i}\left(\tau_{2}+\tau\right) \operatorname{Tr} \sigma^{(2)}+\cdots
\end{aligned}
$$

because both adjacent nodes provide fundamental matter for the dualized node, and hence both FI parameters $\tau_{1}$ and $\tau_{2}$ get shifted by $\tau$.

In the third possibility, we dualize a node that has only anti-fundamental matter as shown in Fig. 7. In this case the 
(A)

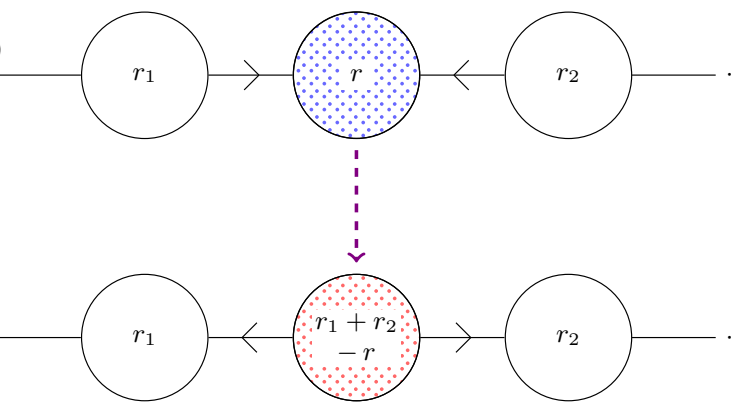

Fig. 7 2d Seiberg duality on a node with only anti-chiral fundamental matter realized by adjacent $2 \mathrm{~d}$ gauge nodes. There are no mesonic fields introduced in this case

classical superpotential before the duality is given again by (38), but after the duality it becomes

$$
\begin{aligned}
\mathcal{W}_{\mathrm{cl}}^{\mathrm{B}}= & \cdots+2 \pi \mathrm{i} \tau_{1} \operatorname{Tr} \sigma^{(1)}-2 \pi \mathrm{i} \tau \operatorname{Tr} \sigma^{\prime} \\
& +2 \pi \mathrm{i} \tau_{2} \operatorname{Tr} \sigma^{(2)}+\cdots
\end{aligned}
$$

with no shifts in $\tau_{1}$ and $\tau_{2}$ since the dualized node has no fundamental matter.

\section{Relating quivers and contours}

In this section we discuss different $2 \mathrm{~d} / 4 \mathrm{~d}$ theories related by Seiberg duality to the oriented quiver represented in Fig. 2. To any of these theories we can associate a system of twisted chiral ring equations that are distinct from the ones we have discussed in Sect. 2.3. However, being related by Seiberg duality, there is a simple one-to-one map among them and their solutions. Then, a natural question arises: how is this duality map reflected on the localization side?

To answer this question, consider again the oriented quiver of Fig. 2, which we now denote by $Q_{0}$. From it we can generate equivalent quivers by dualizing any of the $2 \mathrm{~d}$ nodes. We first carry out a very specific sequence of dualities that are shown in Fig. 8: at each step of the duality chain, the node being dualized has only fundamental matter. Therefore, Seiberg duality always acts as in (40). ${ }^{5}$

For each quiver in the chain, we can proceed as we did in Sect. 2.3 for $Q_{0}$. We integrate out the matter multiplets to obtain the effective twisted chiral superpotential, derive from it the twisted chiral ring equations, solve them about a particular massive vacuum order by order in the strong coupling scales, evaluate the superpotential on the corresponding vacuum and finally compare the result with the ramified instanton calculation with a specific integration contour for the $\chi_{I}$ variables. In this program, the choice of the classical

\footnotetext{
5 The same sequence of dualities has also been mentioned in [7].
}

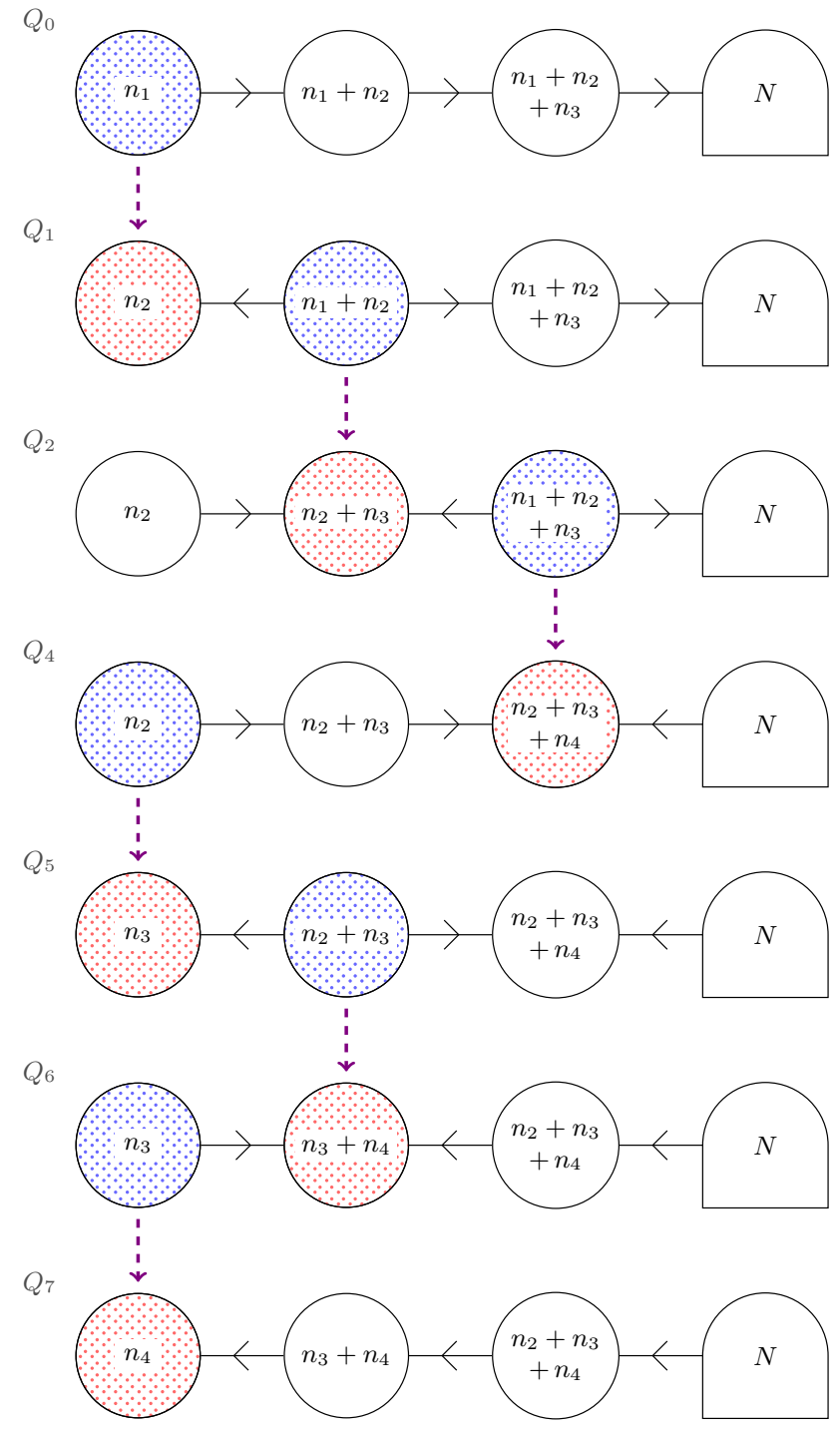

Fig. 8 A sequence of Seiberg dualities obtained by dualizing the node that has only fundamental flavours attached to it at each step. The node that is dualized is indicated by the blue arrow. The reason why in the list of names for the quivers we skipped $Q_{3}$ will become clear later on

vacuum is the first important piece of information which we have to provide.

\section{Classical vacuum}

The classical twisted superpotential for the quiver $Q_{0}$ is

$\mathcal{W}_{\mathrm{cl}}^{Q_{0}}=2 \pi \mathrm{i} \tau_{1} \operatorname{Tr} \sigma^{(1)}+2 \pi \mathrm{i} \tau_{2} \operatorname{Tr} \sigma^{(2)}+2 \pi \mathrm{i} \tau_{3} \operatorname{Tr} \sigma^{(3)}$.

Applying to it the duality rule (40), we obtain the classical superpotential for the quiver $Q_{1}$. With a further duality we obtain the classical superpotential for the quiver $Q_{2}$ and so on along the duality chain of Fig. 8. 
Explicitly these superpotentials are ${ }^{6}$ :

$$
\begin{aligned}
\mathcal{W}_{\mathrm{cl}}^{Q_{1}}= & -2 \pi \mathrm{i} \tau_{1} \operatorname{Tr} \sigma^{(1)}+2 \pi \mathrm{i}\left(\tau_{1}+\tau_{2}\right) \operatorname{Tr} \sigma^{(2)} \\
& +2 \pi \mathrm{i} \tau_{3} \operatorname{Tr} \sigma^{(3)}, \\
\mathcal{W}_{\mathrm{cl}}^{Q_{2}}= & 2 \pi \mathrm{i} \tau_{2} \operatorname{Tr} \sigma^{(1)}-2 \pi \mathrm{i}\left(\tau_{1}+\tau_{2}\right) \operatorname{Tr} \sigma^{(2)} \\
& +2 \pi \mathrm{i}\left(\tau_{1}+\tau_{2}+\tau_{3}\right) \operatorname{Tr} \sigma^{(3)}, \\
\mathcal{W}_{\mathrm{cl}}^{Q_{4}}= & 2 \pi \mathrm{i} \tau_{2} \operatorname{Tr} \sigma^{(1)}+2 \pi \mathrm{i} \tau_{3} \operatorname{Tr} \sigma^{(2)} \\
& -2 \pi \mathrm{i}\left(\tau_{1}+\tau_{2}+\tau_{3}\right) \operatorname{Tr} \sigma^{(3)}, \\
\mathcal{W}_{\mathrm{cl}}^{Q_{5}}= & -2 \pi \mathrm{i} \tau_{2} \operatorname{Tr} \sigma^{(1)}+2 \pi \mathrm{i}\left(\tau_{2}+\tau_{3}\right) \operatorname{Tr} \sigma^{(2)} \\
& -2 \pi \mathrm{i}\left(\tau_{1}+\tau_{2}+\tau_{3}\right) \operatorname{Tr} \sigma^{(3)}, \\
\mathcal{W}_{\mathrm{cl}}^{Q_{6}}= & 2 \pi \mathrm{i} \tau_{3} \operatorname{Tr} \sigma^{(1)}-2 \pi \mathrm{i}\left(\tau_{2}+\tau_{3}\right) \operatorname{Tr} \sigma^{(2)} \\
& -2 \pi \mathrm{i} \tau_{1} \operatorname{Tr} \sigma^{(3)}, \\
\mathcal{W}_{\mathrm{cl}}^{Q_{7}}= & -2 \pi \mathrm{i} \tau_{3} \operatorname{Tr} \sigma^{(1)}-2 \pi \mathrm{i} \tau_{2} \operatorname{Tr} \sigma^{(2)} \\
& -2 \pi \mathrm{i} \tau_{1} \operatorname{Tr} \sigma^{(3)} .
\end{aligned}
$$

From these expressions we can read the map between the FI parameters of any quiver and those of the initial quiver $Q_{0}$. For example, for $Q_{1}$ we have

$\tau_{1}^{Q_{1}}=-\tau_{1}, \quad \tau_{2}^{Q_{1}}=\tau_{1}+\tau_{2}, \quad \tau_{3}^{Q_{1}}=\tau_{3}$,

while for the quiver $Q_{2}$ we have

$\tau_{1}^{Q_{2}}=\tau_{2}, \quad \tau_{2}^{Q_{2}}=-\tau_{1}-\tau_{2}, \tau_{3}^{Q_{2}}=\tau_{1}+\tau_{2}+\tau_{3}$.

The next step is to identify the classical vacuum for each quiver. We already know that for $Q_{0}$ the vacuum that respects the partition $\left[n_{1}, \ldots, n_{4}\right]$ associated to the surface operator, is (see (21))

$\sigma_{\mathrm{cl}}^{(1)}=\mathcal{A}_{1}, \quad \sigma_{\mathrm{cl}}^{(2)}=\mathcal{A}_{1} \oplus \mathcal{A}_{2}, \quad \sigma_{\mathrm{cl}}^{(3)}=\mathcal{A}_{1} \oplus \mathcal{A}_{2} \oplus \mathcal{A}_{3}$.

Since Seiberg duality is an exact infrared equivalence, the classical superpotentials of two dual quivers, evaluated in the respective vacua, should be identical. This requirement immediately fixes the structure of the classical vacuum for all quivers. For instance, for $Q_{1}$ one can check that

$\sigma_{\mathrm{cl}}^{(1)}=\mathcal{A}_{2}, \quad \sigma_{\mathrm{cl}}^{(2)}=\mathcal{A}_{1} \oplus \mathcal{A}_{2}, \quad \sigma_{\mathrm{cl}}^{(3)}=\mathcal{A}_{1} \oplus \mathcal{A}_{2} \oplus \mathcal{A}_{3}$,

leads to the desired match; indeed

$$
\begin{aligned}
\mathcal{W}_{\mathrm{cl}}^{Q_{1}}= & -2 \pi \mathrm{i} \tau_{1} \operatorname{Tr} \mathcal{A}_{2}+2 \pi \mathrm{i}\left(\tau_{1}+\tau_{2}\right)\left(\operatorname{Tr} \mathcal{A}_{1}+\operatorname{Tr} \mathcal{A}_{2}\right) \\
& +2 \pi \mathrm{i} \tau_{3}\left(\operatorname{Tr} \mathcal{A}_{1}+\operatorname{Tr} \mathcal{A}_{2}+\operatorname{Tr} \mathcal{A}_{3}\right)
\end{aligned}
$$

${ }^{6}$ For ease of notation we use the same symbol $\sigma^{(I)}$ to denote the chiral superfield before and after the duality.
Table 1 For each of the quivers in Fig. 8, we list the classical expectation values of the twisted chiral fields in each of the three $2 \mathrm{~d}$ nodes. Using them in the classical twisted chiral superpotentials given in (43), one finds identical expressions

\begin{tabular}{llll}
\hline Quiver & $\sigma_{\mathrm{cl}}^{(1)}$ & $\sigma_{\mathrm{cl}}^{(2)}$ & $\sigma_{\mathrm{cl}}^{(3)}$ \\
\hline$Q_{0}$ & $\mathcal{A}_{1}$ & $\mathcal{A}_{1} \oplus \mathcal{A}_{2}$ & $\mathcal{A}_{1} \oplus \mathcal{A}_{2} \oplus \mathcal{A}_{3}$ \\
$Q_{1}$ & $\mathcal{A}_{2}$ & $\mathcal{A}_{1} \oplus \mathcal{A}_{2}$ & $\mathcal{A}_{1} \oplus \mathcal{A}_{2} \oplus \mathcal{A}_{3}$ \\
$Q_{2}$ & $\mathcal{A}_{2}$ & $\mathcal{A}_{2} \oplus \mathcal{A}_{3}$ & $\mathcal{A}_{1} \oplus \mathcal{A}_{2} \oplus \mathcal{A}_{3}$ \\
$Q_{4}$ & $\mathcal{A}_{2}$ & $\mathcal{A}_{2} \oplus \mathcal{A}_{3}$ & $\mathcal{A}_{2} \oplus \mathcal{A}_{3} \oplus \mathcal{A}_{4}$ \\
$Q_{5}$ & $\mathcal{A}_{3}$ & $\mathcal{A}_{2} \oplus \mathcal{A}_{3}$ & $\mathcal{A}_{2} \oplus \mathcal{A}_{3} \oplus \mathcal{A}_{4}$ \\
$Q_{6}$ & $\mathcal{A}_{3}$ & $\mathcal{A}_{3} \oplus \mathcal{A}_{4}$ & $\mathcal{A}_{2} \oplus \mathcal{A}_{3} \oplus \mathcal{A}_{4}$ \\
$Q_{7}$ & $\mathcal{A}_{4}$ & $\mathcal{A}_{3} \oplus \mathcal{A}_{4}$ & $\mathcal{A}_{2} \oplus \mathcal{A}_{3} \oplus \mathcal{A}_{4}$ \\
\hline
\end{tabular}

$$
\begin{aligned}
= & +2 \pi \mathrm{i} \tau_{1} \operatorname{Tr} \mathcal{A}_{1}+2 \pi \mathrm{i} \tau_{2}\left(\operatorname{Tr} \mathcal{A}_{1}+\operatorname{Tr} \mathcal{A}_{2}\right) \\
& +2 \pi \mathrm{i} \tau_{3}\left(\operatorname{Tr} \mathcal{A}_{1}+\operatorname{Tr} \mathcal{A}_{2}+\operatorname{Tr} \mathcal{A}_{3}\right) \\
= & \mathcal{W}_{\mathrm{cl}}^{Q_{0}} .
\end{aligned}
$$

This calculation can be easily generalized to all other quivers in the duality chain and the results are summarized in Table 1.

The $q$ vs $\Lambda$ map

The next necessary ingredient is the relation between the ramified instanton parameters $q_{I}$ and the strong coupling scales $\Lambda_{I}^{Q_{i}}$ of a given quiver.

For the first quiver $Q_{0}$, the $q$ vs $\Lambda$ map was already derived and written in (32). If we now consider the second quiver $Q_{1}$, from the running of the FI parameters we find

$$
\begin{aligned}
\left(\Lambda_{1}^{Q_{1}}\right)^{-n_{1}-n_{2}} & =\mathrm{e}^{2 \pi \mathrm{i} \tau_{1}^{Q_{1}}} \mu^{-n_{1}-n_{2}}, \\
\left(\Lambda_{2}^{Q_{1}}\right)^{n_{1}+2 n_{2}+n_{3}} & =\mathrm{e}^{2 \pi \mathrm{i} \tau_{2}^{Q_{1}}} \mu^{n_{1}+2 n_{2}+n_{3}}, \\
\left(\Lambda_{3}^{Q_{1}}\right)^{n_{3}+n_{4}} & =\mathrm{e}^{2 \pi \mathrm{i} \tau_{3}^{Q_{1}}} \mu^{n_{3}+n_{4}} .
\end{aligned}
$$

Using the relations (49) and the definitions (33), it is easy to obtain (up to inessential signs) the $q$ vs $\Lambda$ map in this case, namely

$$
\begin{aligned}
& q_{1} \sim\left(\Lambda_{1}^{Q_{1}}\right)^{n_{1}+n_{2}}, \\
& q_{2} \sim \frac{\left(\Lambda_{2}^{Q_{1}}\right)^{n_{1}+2 n_{2}+n_{3}}}{\left(\Lambda_{1}^{Q_{1}}\right)^{n_{1}+n_{2}}}, \\
& q_{3} \sim\left(\Lambda_{3}^{Q_{1}}\right)^{n_{3}+n_{4}} .
\end{aligned}
$$

Applying the same procedure to $Q_{2}$ and using (50), we find

$$
\begin{aligned}
& q_{1} \sim \frac{\left(\Lambda_{2}^{Q_{2}}\right)^{n_{1}+2 n_{2}+n_{3}}}{\left(\Lambda_{1}^{Q_{2}}\right)^{n_{2}+n_{3}}}, \\
& q_{2} \sim\left(\Lambda_{1}^{Q_{2}}\right)^{n_{2}+n_{3}}, \\
& q_{3} \sim \frac{\left(\Lambda_{3}^{Q_{2}}\right)^{N+n_{2}+n_{3}}}{\left(\Lambda_{2}^{Q_{2}}\right)^{n_{1}+2 n_{2}+n_{3}}} .
\end{aligned}
$$


Table 2 For each quiver of Fig. 8, we list the $q$ vs $\Lambda$ map (up to sign factors, which can be found in Appendix B). The exponent of each strong coupling scale is determined by the number of effective flavours at that node in the quiver and is related to the $\beta$-function coefficient of the corresponding FI parameter

\begin{tabular}{llll}
\hline Quiver & $q_{1}$ & $q_{2}$ & $q_{3}$ \\
\hline$Q_{0}$ & $\Lambda_{1}^{n_{1}+n_{2}}$ & $\Lambda_{2}^{n_{2}+n_{3}}$ & $\Lambda_{3}^{n_{3}+n_{4}}$ \\
$Q_{1}$ & $\left(\Lambda_{1}^{Q_{1}}\right)^{n_{1}+n_{2}}$ & $\frac{\left(\Lambda_{2}^{Q_{1}}\right)^{n_{1}+2 n_{2}+n_{3}}}{\left(\Lambda_{1}^{Q_{1}}\right)^{n_{1}+n_{2}}}$ & $\left(\Lambda_{3}^{Q_{1}}\right)^{n_{3}+n_{4}}$ \\
$Q_{2}$ & $\frac{\left(\Lambda_{2}^{Q_{2}}\right)^{n_{1}+2 n_{2}+n_{3}}}{\left(\Lambda_{1}^{Q_{2}}\right)^{n_{2}+n_{3}}}$ & $\left(\Lambda_{1}^{Q_{2}}\right)^{n_{2}+n_{3}}$ & $\frac{\left(\Lambda_{3}^{Q_{2}}\right)^{N+n_{2}+n_{3}}}{\left(\Lambda_{2}^{Q_{2}}\right)^{n_{1+2 n_{2}+n_{3}}}}$ \\
$Q_{4}$ & $\frac{\left(\Lambda_{3}^{Q_{4}}\right)^{N+n_{2}+n_{3}}}{\left(\Lambda_{1}^{Q_{4}}\right)^{n_{2}+n_{3}}\left(\Lambda_{2}^{Q_{4}}\right)^{n_{3}+n_{4}}}$ & $\left(\Lambda_{1}^{Q_{4}}\right)^{n_{2}+n_{3}}$ & $\left(\Lambda_{2}^{Q_{4}}\right)^{n_{3}+n_{4}}$ \\
$Q_{5}$ & $\frac{\left(\Lambda_{3}^{Q_{5}}\right)^{N+n_{2}+n_{3}}}{\left(\Lambda_{2}^{Q_{5}}\right)^{n_{2}+2 n_{3}+n_{4}}}$ & $\left(\Lambda_{1}^{Q_{5}}\right)^{n_{2}+n_{3}}$ & $\frac{\left(\Lambda_{2}^{Q_{5}}\right)^{n_{2}+2 n_{3}+n_{4}}}{\left(\Lambda_{1}^{Q_{5}}\right)^{n_{2}+n_{3}}}$ \\
$Q_{6}$ & $\left(\Lambda_{3}^{Q_{6}}\right)^{n_{1}+n_{2}}$ & $\left(\Lambda_{2}^{Q_{6}}\right)^{n_{2}+2 n_{3}+n_{4}}$ & $\left(\Lambda_{1}^{Q_{6}}\right)^{n_{3}+n_{4}}$ \\
$Q_{7}$ & $\left(\Lambda_{3}^{Q_{7}}\right)^{n_{1}+n_{2}}$ & $\left(\Lambda_{2}^{Q_{7}}\right)^{n_{2}+n_{3}}$ & $\left(\Lambda_{1}^{Q_{7}}\right)^{n_{3}+n_{4}}$ \\
\hline
\end{tabular}

Repeating this analysis for all quivers of Fig. 8, we obtain the results collected in Table 2 .

We finally recall that the following relation

$q_{4} \sim \frac{\Lambda_{4 \mathrm{~d}}^{2 N}}{q_{1} q_{2} q_{3}}$

holds for all quivers.

From Table 2, we observe that except for the oriented quivers $Q_{0}$ and $Q_{7}$, in all other cases the contributions of a single ramified instanton can be proportional to a ratio of strong coupling scales. It would be interesting to understand the origin of this fact from the perspective of vortex solutions in $2 \mathrm{~d}$ quivers with bi-fundamental matter. However, for our present purposes it is important to keep in mind that the ramified instanton partition function is a power series in $q_{I}$. This means that, except for the quivers $Q_{0}$ and $Q_{7}$, we are forced to have some hierarchy among the scales $\Lambda_{I}^{Q_{i}}$ in order for the $q$ vs $\Lambda$ map to be consistent with the power series expansion of the ramified instanton partition function. For instance for the quiver $Q_{1}$, we see from Table 2 that if we want that both $q_{1}$ and $q_{2}$ be "small", it is necessary to have

$1>\left|\frac{\Lambda_{1}^{Q_{1}}}{\mu}\right|^{n_{1}+n_{2}} \gg\left|\frac{\Lambda_{2}^{Q_{1}}}{\mu}\right|^{n_{1}+2 n_{2}+n_{3}}$.

Using (54) and the fact that the $\beta$-function coefficient of the first node is negative, we can easily see that (58) is equivalent to

$0<-\zeta_{1}^{Q_{1}} \ll \zeta_{2}^{Q_{1}}$
Notice that this inequality follows from the duality relations (49): indeed, $\zeta_{1}^{Q_{1}}=-\zeta_{1}$ and $\zeta_{2}^{Q_{1}}=\zeta_{1}+\zeta_{2}$, with $\zeta_{I}>0$ as indicated in (19).

In a similar way, for quiver $Q_{2}$ we see from Table 2 that in order for the instanton weights $q_{I}$ to be "small", we must have

$1>\left|\frac{\Lambda_{1}^{Q_{2}}}{\mu}\right|^{n_{2}+n_{3}} \gg\left|\frac{\Lambda_{2}^{Q_{2}}}{\mu}\right|^{n_{1}+2 n_{2}+n_{3}} \gg\left|\frac{\Lambda_{3}^{Q_{2}}}{\mu}\right|^{N+n_{2}+n_{3}}$,

which, taking into account the signs of the $\beta$-function coefficients, in this case implies that

$0<\zeta_{1}^{Q_{2}} \ll-\zeta_{2}^{Q_{2}} \ll \zeta_{3}^{Q_{2}}$

Again we can check that this hierarchy just follows from the duality relations (50), since $\zeta_{1}^{Q_{2}}=\zeta_{2}, \zeta_{2}^{Q_{2}}=-\zeta_{1}-\zeta_{2}$ and $\zeta_{3}^{Q_{2}}=\zeta_{1}+\zeta_{2}+\zeta_{3}$, with $\zeta_{I}>0$.

We can repeat this analysis for all linear quivers of the sequence, and always find the same pattern: when a hierarchy of scales is needed in order to have a meaningful ramified instanton expansion, this is automatically guaranteed by the duality relations among the real FI parameters of the various quivers. Moreover, the $4 \mathrm{~d}$ low-energy scale $\Lambda_{4 \mathrm{~d}}$ is always the smallest scale in view of (57).

\subsection{Contour prescriptions for dual quivers}

We now address the question of how the non-perturbative superpotential associated to each quiver can be obtained from the ramified instanton partition function (8) using a suitable contour prescription for the $\chi_{I}$-integrals. In Sect. 2.3 we answered this question for the oriented quiver $Q_{0}$ by comparing each term of the solution of the chiral ring equations with the localization results. Here we provide a general argument that allows one to derive the appropriate contour prescription for any quiver of the duality chain, without explicitly solving the twisted chiral ring equations and integrating them in. We perform a detailed analysis at the one-instanton level, but our conclusions are valid also at higher instantons.

Let us first consider only the three $2 \mathrm{~d}$ nodes and neglect for the moment the contribution of the $4 \mathrm{~d}$ node by setting $\Lambda_{4 \mathrm{~d}} \rightarrow 0$ and hence, according to (57), $q_{4} \rightarrow 0$. Using the partition function (31), the one-instanton superpotential in this case can be written as

$$
\mathcal{W}_{1 \text {-inst }}=\sum_{I=1}^{3} q_{I} w_{I}
$$


where

$$
\begin{aligned}
w_{I}= & -\lim _{\epsilon_{1}, \hat{\epsilon}_{2} \rightarrow 0} \int \frac{d \chi_{I}}{2 \pi \mathrm{i}} \prod_{s \in \mathcal{N}_{I}} \frac{1}{\left(a_{s}-\chi_{I}+\frac{1}{2}\left(\epsilon_{1}+\hat{\epsilon}_{2}\right)\right)} \\
& \times \prod_{t \in \mathcal{N}_{I+1}} \frac{1}{\left(\chi_{I}-a_{t}+\frac{1}{2}\left(\epsilon_{1}+\hat{\epsilon}_{2}\right)\right)} .
\end{aligned}
$$

From this we immediately see that $w_{I}$ can have either $n_{I}$ or $n_{I+1}$ terms depending on whether the $\chi_{I}$-contour is closed in the upper or lower half plane, respectively. On the other hand, exploiting the relation [10]

$\operatorname{Tr} \sigma_{\star}^{(I)}=\operatorname{Tr}\left(\sigma_{\mathrm{cl}}^{(I)}+\delta \sigma^{(I)}\right)=\left.\frac{1}{b_{I}} \Lambda_{I} \frac{\partial \mathcal{W}}{\partial \Lambda_{I}}\right|_{\sigma_{\star}}$,

and the maps in Table 2, we can understand which type of ramified instantons contributes to each term proportional to $\operatorname{Tr} \sigma^{(I)}$. For example, for $Q_{0}$ using the map (32), we find

$\operatorname{Tr} \delta \sigma^{(1)}=q_{1} w_{1}$,
$\operatorname{Tr} \delta \sigma^{(2)}=q_{2} w_{2}$,
$\operatorname{Tr} \delta \sigma^{(3)}=q_{3} w_{3}$.

These relations establish a natural correspondence between the nodes of the quiver and the instanton counting parameters $q_{I}$ and the corresponding $\chi_{I}$ fields for $I=1,2,3$ : indeed, the first node is associated to $\chi_{1}$, the second node to $\chi_{2}$ and the third node to $\chi_{3}$. Furthermore, exploiting the fact that $\delta \sigma^{(I)}$ must have the same structure of the classical part $\sigma_{\mathrm{cl}}^{(I)}$ and hence that their entries can only arise in any of the blocks that make up the rank of the corresponding $2 \mathrm{~d}$ gauge node, we conclude that we have to close the integration contour in the upper-half plane for all $\chi_{I}$, so that $\operatorname{Tr} \delta \sigma^{(1)}$ has $n_{1}$ contributions, $\operatorname{Tr} \delta \sigma^{(2)}$ has $n_{2}$ contributions and $\operatorname{Tr} \delta \sigma^{(3)}$ has $n_{3}$ contributions. We indicate this choice of integration contour with the notation $\left(\left.\chi_{1}\right|_{+},\left.\chi_{2}\right|_{+},\left.\chi_{3}\right|_{+}\right)$. In this way we have retrieved the same contour prescription of Sect. 2.3, without explicitly solving the twisted chiral ring equations.

The same strategy can be used for the other quivers of the duality chain. Let us consider for example $Q_{1}$. From (64) and the map (55), we find

$$
\begin{aligned}
& \operatorname{Tr} \delta \sigma^{(1)}=q_{1} w_{1}-q_{2} w_{2}, \\
& \operatorname{Tr} \delta \sigma^{(2)}=q_{2} w_{2}, \\
& \operatorname{Tr} \delta \sigma^{(3)}=q_{3} w_{3} .
\end{aligned}
$$

In this case, the correspondence between the second node and $\chi_{2}$ and between the third node and $\chi_{3}$ is again obvious, but since now there are two $w_{I}$ contributing to the first trace, we need to use the hierarchy of scales (58) to disentangle the linear combination. In particular we see that the contribution proportional to $q_{2}$ is sub-dominant and thus can be neglected at leading order. This allows us to conclude that the first node must be unambiguously associated to $\chi_{1}$. However, the number of terms contributing to $\operatorname{Tr} \delta \sigma^{(1)}$ must be $n_{2}$, since for $Q_{1}$ we have $\sigma_{\mathrm{cl}}^{(1)}=\mathcal{A}_{2}$ (see (52)). Thus, the $\chi_{1}$-integral should be closed in the lower half-plane to provide this number of terms, while the integrations over $\chi_{2}$ and $\chi_{3}$ must be carried out in the upper half-plane as before. In conclusion, to $Q_{1}$ we assign the contour prescription $\left(\left.\chi_{1}\right|_{-},\left.\chi_{2}\right|_{+},\left.\chi_{3}\right|_{+}\right)$. It can be checked that with this choice the localization results perfectly agree, term by term, with the solution of the appropriate chiral ring equations (see Appendix B for details).

Comparing $\mathcal{W}_{\mathrm{cl}}^{Q_{0}}$ and $\mathcal{W}_{\mathrm{cl}}^{Q_{1}}$ given in (42) and (43), we notice that an indication for the flipping of the $\chi_{1}$ integration contour between $Q_{0}$ and $Q_{1}$ can be traced to the change in sign of the term containing $\operatorname{Tr} \sigma^{(1)}$, or equivalently to the change in sign of the $\beta$-function coefficient and of the FI parameter of the first node under the duality map from $Q_{0}$ to $Q_{1}$. We propose that this is in fact the rule, and that it is the sign of the $\beta$-function coefficient for a given node (or of its FI parameter) that determines whether the contour of integration for the corresponding $\chi$ variable has to be closed in the upper or in the lower half-plane.

As a simple and non-trivial check of this proposal we consider the quiver $Q_{2}$. Here, using the $q$ vs $\Lambda$ map of Table 2 into (64), we find

$$
\begin{aligned}
& \operatorname{Tr} \delta \sigma^{(1)}=q_{2} w_{2}-q_{1} w_{1}, \\
& \operatorname{Tr} \delta \sigma^{(2)}=q_{1} w_{1}, \\
& \operatorname{Tr} \delta \sigma^{(3)}=q_{3} w_{3} .
\end{aligned}
$$

From the second and third relations respectively, we see that $\chi_{1}$ is associated to the second node and $\chi_{3}$ to the third node. To decide which $\chi$-variable is associated to the first node, we again exploit the hierarchy of scales (60), which for the case at hand implies that $q_{1}$ is sub-dominant with respect to $q_{2}$. Thus, the $q_{1}$-term in the first relation of (50) can be neglected at leading order, implying that $\chi_{2}$ must be associated to the first node. Notice that it is the second node of $Q_{2}$ that has a negative $\beta$-function, and hence a negative FI parameter, and so it is again $\chi_{1}$ that has to be integrated in the lower half-plane. We then conclude that to the quiver $Q_{2}$ we must assign the contour prescription $\left(\left.\chi_{2}\right|_{+},\left.\chi_{1}\right|_{-},\left.\chi_{3}\right|_{+}\right)$. A similar analysis can be done for all other quivers of the sequence in Fig. 8.

Let us now turn to the contour for the last integration variable $\chi_{4}$. To specify it, we have to switch on the dynamics on the $4 \mathrm{~d}$ node of the quiver, since the corresponding parameter $q_{4}$ is non-zero only when $\Lambda_{4 \mathrm{~d}}$ is non-zero (see (57)). Thus, $q_{4}$ and hence $\chi_{4}$ cannot be associated to any of the $2 \mathrm{~d}$ nodes and must be related to the $4 \mathrm{~d}$ node. By observing the duality chain, we see that the third node, which is the only $2 \mathrm{~d}$ node connected to the $4 \mathrm{~d}$ node, is dualized precisely once. Until this point the $4 \mathrm{~d}$ node provides fundamental matter to the third $2 \mathrm{~d}$ node, while from this point on it provides antifundamental matter. Given that we know that for the initial quiver $Q_{0}$ the variable $\chi_{4}$ has to be integrated in the lower 
Table 3 For each quiver $Q_{i}$ in Fig. 8, we list the signs of the $\beta$-function coefficients $b_{I}^{Q_{i}}$ for the three $2 \mathrm{~d}$ nodes, which are also the signs of the corresponding FI parameters $\zeta_{I}^{Q_{i}}$. These signs determine whether the integration contour for the corresponding $\chi$-variable has to be closed in the upper $(+)$ or lower (-) half-plane. The last column displays the contour prescription from which we can also read which $\chi$-variable is associated to which node of the quiver. The variable $\chi_{4}$ is always the last one to be integrated

\begin{tabular}{lllll}
\hline Quiver & $\operatorname{sgn}\left(b_{1}^{Q_{i}}\right)$ & $\operatorname{sgn}\left(b_{2}^{Q_{i}}\right)$ & $\operatorname{sgn}\left(b_{3}^{Q_{i}}\right)$ & Contour prescription \\
\hline$Q_{0}$ & + & + & + & $\left(\left.\chi_{1}\right|_{+},\left.\chi_{2}\right|_{+},\left.\chi_{3}\right|_{+},\left.\chi_{4}\right|_{-}\right)$ \\
$Q_{1}$ & - & + & + & $\left(\left.\chi_{1}\right|_{-},\left.\chi_{2}\right|_{+},\left.\chi_{3}\right|_{+},\left.\chi_{4}\right|_{-}\right)$ \\
$Q_{2}$ & + & - & + & $\left(\left.\chi_{2}\right|_{+},\left.\chi_{1}\right|_{-},\left.\chi_{3}\right|_{+},\left.\chi_{4}\right|_{-}\right)$ \\
$Q_{4}$ & + & + & - & $\left(\left.\chi_{2}\right|_{+},\left.\chi_{3}\right|_{+},\left.\chi_{1}\right|_{-},\left.\chi_{4}\right|_{+}\right)$ \\
$Q_{5}$ & - & + & - & $\left(\left.\chi_{2}\right|_{-},\left.\chi_{3}\right|_{+},\left.\chi_{1}\right|_{-},\left.\chi_{4}\right|_{+}\right)$ \\
$Q_{6}$ & + & - & - & $\left(\left.\chi_{3}\right|_{+},\left.\chi_{2}\right|_{-},\left.\chi_{1}\right|_{-},\left.\chi_{4}\right|_{+}\right)$ \\
$Q_{7}$ & - & - & - & $\left(\left.\chi_{3}\right|_{-},\left.\chi_{2}\right|_{-},\left.\chi_{1}\right|_{-},\left.\chi_{4}\right|_{+}\right)$ \\
\hline
\end{tabular}

half-plane, we are naturally led to propose that the contour for $\chi_{4}$ remains in the lower plane (-) until the third node is dualized, i.e. for $Q_{0}, Q_{1}$ and $Q_{2}$, and then it flips to the upper half-plane $(+)$, remaining unchanged for the rest of the duality chain, i.e. for $Q_{4}, Q_{5}, Q_{6}$ and $Q_{7}$. We have verified the validity of this proposal by explicitly solving the twisted chiral ring equations for all seven quivers to obtain the corresponding twisted superpotentials, and checking that these agree term by term with what the ramified instanton partition function yields with the proposed integration prescriptions (see Appendix B for details). Our results on the contour assignments for the various quivers are summarized in Table 3. ${ }^{7}$

\subsection{The Jeffrey-Kirwan prescription for dual quivers}

At one-instanton it is sufficient to specify whether the contours of integration for $\chi_{I}$ are closed in the upper or lower half-planes to completely specify the prescription. However, at higher instantons this may be no longer sufficient since also the order in which the integrations are performed may become relevant to have a one-to-one correspondence between the terms appearing in the superpotential derived from the twisted chiral ring equations and the residues contributing in the localization integrals.

An elegant way to fully specify the contour of integration for all variables (including the order in which they are integrated) is using the Jeffrey-Kirwan (JK) residue prescription [20] (see also, for example, [7,31,32] for recent applications to gauge theories). The essential point of this prescription

\footnotetext{
$\overline{7}$ We remark that the results for the last quiver $Q_{7}$ coincide with those derived in Ref. [10], once the nodes are numbered in the opposite order.
}

is that the set of poles chosen by a contour is completely specified by the so-called JK reference vector $\eta$.

As we have seen before, for the oriented quiver $Q_{0}$ the variable $\chi_{4}$ associated to the $4 \mathrm{~d}$ gauge node has to be integrated as the last one in the lower-half plane, while the variables $\chi_{1}, \chi_{2}$ and $\chi_{3}$, associated to the first, second and third node respectively, have to be integrated in the upper-half plane but no particular order of integration is required in this case. This means that the JK vector for the quiver $Q_{0}$ can be written as

$\eta_{Q_{0}}=-\zeta_{1} \chi_{1}-\zeta_{2} \chi_{2}-\zeta_{3} \chi_{3}+\zeta_{4} \chi_{4}$

where $\zeta_{I}$, with $I=1,2,3$, are the FI parameters of the three $2 \mathrm{~d}$ nodes of the quiver and $\zeta_{4}$ is a positive real number such that

$\zeta_{4} \gg \zeta_{I}$

for $I=1,2,3$. As remarked in (19), the FI parameters are positive, so that, given our sign conventions, the vector (68) indeed selects a contour in the upper-half plane for $\chi_{I}$ with $I=1,2,3$. The JK prescription corresponding to (68) requires that these integrals are successively performed according to the magnitudes of $\zeta_{I}$. However, the order of integration does not affect the final result, and thus this prescription always gives the correct answer no matter how the FI parameters are ordered. The inequality (69) implies, instead, that the integral over $\chi_{4}$ is the last one to be performed and, because of the + sign in the last term of $\eta_{Q_{0}}$, this integral must be computed along a contour in the lower-half plane.

Let us now consider the quiver $Q_{1}$. In this case, the JK vector that selects the appropriate contour of integration can be written as

$$
\begin{aligned}
\eta_{Q_{1}} & =+\left|\zeta_{1}^{Q_{1}}\right| \chi_{1}-\left|\zeta_{2}^{Q_{1}}\right| \chi_{2}-\left|\zeta_{3}^{Q_{1}}\right| \chi_{3}+\zeta_{4} \chi_{4} \\
& =-\zeta_{1}^{Q_{1}} \chi_{1}-\zeta_{2}^{Q_{1}} \chi_{2}-\zeta_{3}^{Q_{1}} \chi_{3}+\zeta_{4} \chi_{4}
\end{aligned}
$$

where $\zeta_{I}^{Q_{1}}$, with $I=1,2,3$, are the FI parameters of the the three $2 \mathrm{~d}$ nodes of $Q_{1}$ and $\zeta_{4}$ is a positive number such that

$\zeta_{4} \gg\left|\zeta_{I}^{Q_{1}}\right|$.

We recall that in the quiver $Q_{1}$ the FI parameters satisfy the inequality (59). Consequently, the JK vector (70) implies that the integral over $\chi_{1}$ must be computed in the lowerhalf plane before the integral over $\chi_{2}$, which instead must be computed along a contour in the upper-half plane. The last integral is the one over $\chi_{4}$ which must be computed along a contour in the lower half-plane. The order of integration over $\chi_{1}$ and $\chi_{2}$ is crucial at higher instantons to achieve a oneto-one correspondence between the superpotential obtained from the chiral ring equations and the one computed using the ramified instantons. Some details on this fact at the twoinstanton level are provided in Appendix C. 
Table 4 For each quiver we list the JK reference vector that picks the appropriate contour on the localization side. The parameter $\zeta_{4}$ is always positive and bigger in magnitude than any of the FI parameters. If $\zeta_{I}^{Q_{i}}>0$ the associated $\chi$-variable is integrated along a contour in the upper-half plane, while if $\zeta_{I}^{Q_{i}}<0$ it is integrated in the lower-half plane, in agreement with the prescription in the last column of Table 3

\begin{tabular}{ll}
\hline Quiver & JK vector \\
\hline$Q_{0}$ & $-\zeta_{1} \chi_{1}-\zeta_{2} \chi_{2}-\zeta_{3} \chi_{3}+\zeta_{4} \chi_{4}$ \\
$Q_{1}$ & $-\zeta_{1}^{Q_{1}} \chi_{1}-\zeta_{2}^{Q_{1}} \chi_{2}-\zeta_{3}^{Q_{1}} \chi_{3}+\zeta_{4} \chi_{4}$ \\
$Q_{2}$ & $-\zeta_{1}^{Q_{2}} \chi_{2}-\zeta_{2}^{Q_{2}} \chi_{1}-\zeta_{3}^{Q_{2}} \chi_{3}+\zeta_{4} \chi_{4}$ \\
$Q_{4}$ & $-\zeta_{1}^{Q_{4}} \chi_{2}-\zeta_{2}^{Q_{4}} \chi_{3}-\zeta_{3}^{Q_{4}} \chi_{1}-\zeta_{4} \chi_{4}$ \\
$Q_{5}$ & $-\zeta_{1}^{Q_{5}} \chi_{2}-\zeta_{2}^{Q_{5}} \chi_{3}-\zeta_{3}^{Q_{5}} \chi_{1}-\zeta_{4} \chi_{4}$ \\
$Q_{6}$ & $-\zeta_{1}^{Q_{6}} \chi_{3}-\zeta_{2}^{Q_{6}} \chi_{2}-\zeta_{3}^{Q_{6}} \chi_{1}-\zeta_{4} \chi_{4}$ \\
$Q_{7}$ & $-\zeta_{1}^{Q_{7}} \chi_{3}-\zeta_{2}^{Q_{7}} \chi_{2}-\zeta_{3}^{Q_{7}} \chi_{1}-\zeta_{4} \chi_{4}$ \\
\hline
\end{tabular}

For the quiver $Q_{2}$ one can see that the appropriate integration contour corresponds to the following $\mathrm{JK}$ vector

$$
\begin{aligned}
\eta_{Q_{2}} & =-\left|\zeta_{1}^{Q_{2}}\right| \chi_{2}+\left|\zeta_{2}^{Q_{2}}\right| \chi_{1}-\left|\zeta_{3}^{Q_{2}}\right| \chi_{3}+\zeta_{4} \chi_{4} \\
& =-\zeta_{1}^{Q_{2}} \chi_{2}-\zeta_{2}^{Q_{2}} \chi_{1}-\zeta_{3}^{Q_{2}} \chi_{3}+\zeta_{4} \chi_{4}
\end{aligned}
$$

where the FI parameters satisfy the inequality (61) and the last parameter $\zeta_{4}$ is such that

$\zeta_{4} \gg\left|\zeta_{I}^{Q_{2}}\right|$

Using this, we can see a precise correlation with the prescription $\left(\left.\chi_{2}\right|_{+},\left.\chi_{1}\right|_{-},\left.\chi_{3}\right|_{+},\left.\chi_{4}\right|_{-}\right)$which we discussed above for $Q_{2}$. Notice that in this case the integrals are performed in a specific order, starting form $\chi_{2}$ and finishing with $\chi_{4}$. This order is essential at higher instantons to obtain a perfect match, term by term, between the results from the chiral ring equations and those from localization (see Appendix $\mathrm{C}$ for some details at the two-instanton level).

This procedure can be systematically applied to all quivers in the duality chain of Fig. 8, and the corresponding JK reference vectors are listed in Table $4 .{ }^{8}$

\subsection{New quivers and the corresponding contours}

The chain of Seiberg dualities shown in Fig. 8 is of a very special kind, since the $2 \mathrm{~d}$ gauge node being dualized at each step always has only fundamental flavours attached to it. This ensures that the resulting quivers are always linear. We now relax this condition and consider an alternative duality chain with the same initial and final points, but in which we start by dualizing the second node of the quiver $Q_{0}$ that has both fundamental and anti-fundamental flavours attached to it. This duality leads to the quiver $\widehat{Q}_{1}$ which contains a loop, as shown

\footnotetext{
${ }^{8}$ Similar JK prescriptions have been considered in [33] for quiver theories in a $3 \mathrm{~d}$ context.
}

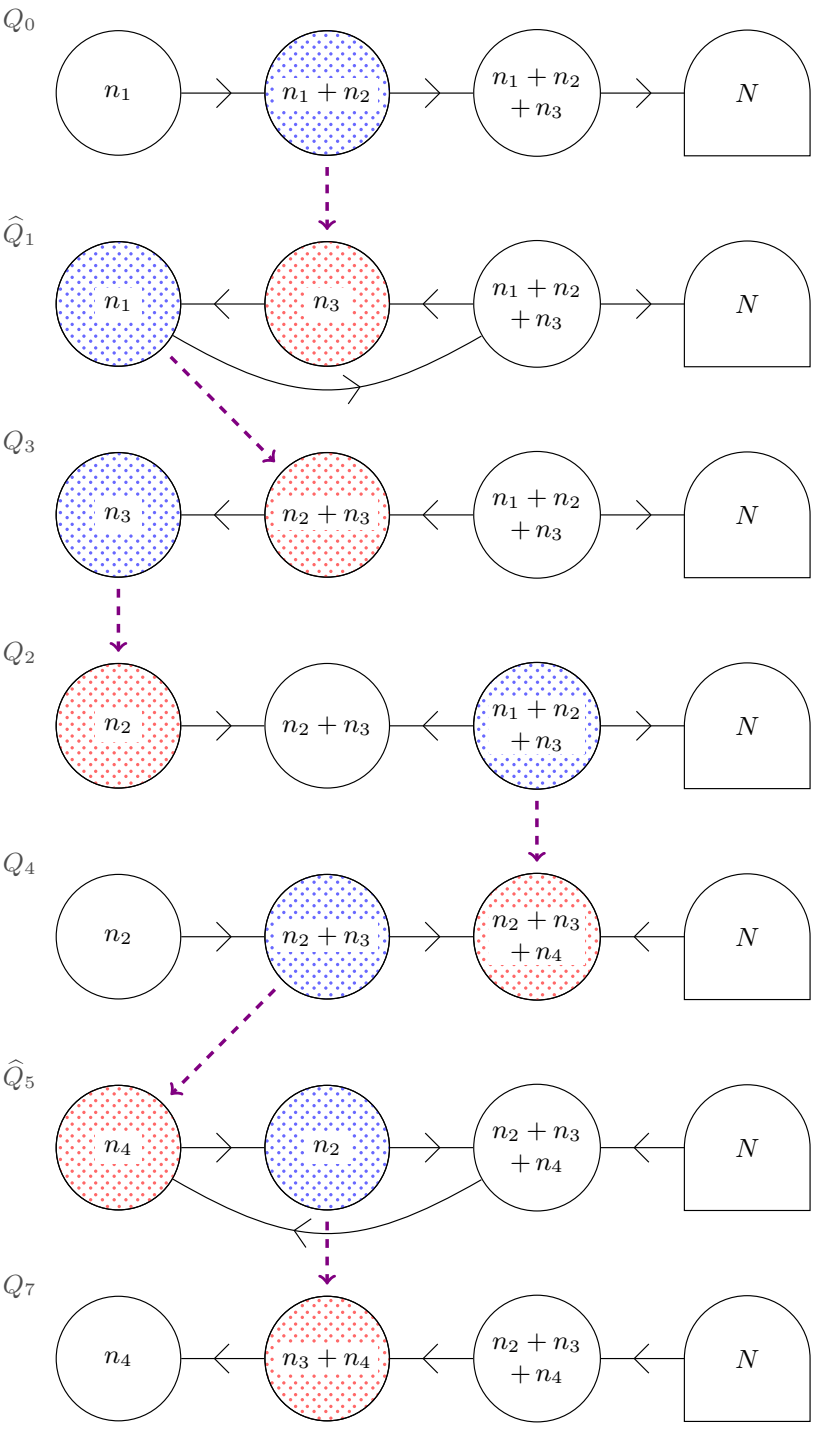

Fig. 9 Another chain of dualities to proceed from $Q_{0}$ to $Q_{7}$

in Fig. 9. Proceeding all the way down as indicated in this figure, we encounter the quivers $Q_{2}$ and $Q_{4}$, which were also part of the earlier sequence, but we also find two new quivers, which we call $Q_{3}$ and $\widehat{Q}_{5}$. The latter, like $\widehat{Q}_{1}$, contains a loop.

We can repeat the same analysis as before and derive the contour prescription for all quivers in this sequence, including the non-linear ones. The first step is obtaining the classical part of the superpotential. Starting from $\mathcal{W}_{\mathrm{cl}}^{Q_{0}}$ given in (42) and applying the duality rule (39) to the second node, we find that the classical part of the superpotential for $\widehat{Q}_{1}$ is

$$
\begin{aligned}
\mathcal{W}_{\mathrm{cl}}^{\widehat{Q}_{1}}= & 2 \pi \mathrm{i} \tau_{1} \operatorname{Tr} \sigma^{(1)}-2 \pi \mathrm{i} \tau_{2} \operatorname{Tr} \sigma^{(2)} \\
& +2 \pi \mathrm{i}\left(\tau_{2}+\tau_{3}\right) \operatorname{Tr} \sigma^{(3)} .
\end{aligned}
$$

If we now dualize the first node of $\widehat{Q}_{1}$ we obtain a new linear quiver $Q_{3}$. Here it is natural to relabel the nodes in 
Table 5 For the quivers $\widehat{Q}_{1}, Q_{3}$ and $\widehat{Q}_{5}$ drawn in Fig. 9, we list the classical expectation values of the twisted chiral fields in each of the $2 \mathrm{~d}$ nodes, about which one finds the solution to the twisted chiral ring. Using this vacuum, along with the FI couplings in the classical twisted chiral superpotentials for each quiver, one finds identical expressions at leading order. The vacuum for the other quivers of the duality chain, namely $Q_{0}, Q_{2}, Q_{4}$ and $Q_{7}$, can be read from Table 1

\begin{tabular}{llll}
\hline Quiver & $\sigma_{\mathrm{cl}}^{(1)}$ & $\sigma_{\mathrm{cl}}^{(2)}$ & $\sigma_{\mathrm{cl}}^{(3)}$ \\
\hline$\widehat{Q}_{1}$ & $\mathcal{A}_{1}$ & $\mathcal{A}_{3}$ & $\mathcal{A}_{1} \oplus \mathcal{A}_{2} \oplus \mathcal{A}_{3}$ \\
$Q_{3}$ & $\mathcal{A}_{3}$ & $\mathcal{A}_{2} \oplus \mathcal{A}_{3}$ & $\mathcal{A}_{1} \oplus \mathcal{A}_{2} \oplus \mathcal{A}_{3}$ \\
$\widehat{Q}_{5}$ & $\mathcal{A}_{4}$ & $\mathcal{A}_{2}$ & $\mathcal{A}_{2} \oplus \mathcal{A}_{3} \oplus \mathcal{A}_{4}$ \\
\hline
\end{tabular}

such a way that the dualized node corresponds to $I=2$, thus respecting the order shown in Fig. 9. Taking this into account and applying the duality map to (74), we then obtain

$$
\begin{aligned}
\mathcal{W}_{\mathrm{cl}}^{Q_{3}}= & -2 \pi \mathrm{i} \tau_{2} \operatorname{Tr} \sigma^{(1)}-2 \pi \mathrm{i} \tau_{1} \operatorname{Tr} \sigma^{(2)} \\
& +2 \pi \mathrm{i}\left(\tau_{1}+\tau_{2}+\tau_{3}\right) \operatorname{Tr} \sigma^{(3)} .
\end{aligned}
$$

In the next two duality steps we find the quivers $Q_{2}$ and $Q_{4}$ whose classical superpotentials are given in (43). Dualizing the second node of $Q_{4}$, we obtain the non-linear quiver $\widehat{Q}_{5}$, whose classical superpotential is

$$
\begin{aligned}
\mathcal{W}_{\mathrm{cl}}^{\widehat{Q}_{5}}= & -2 \pi \mathrm{i} \tau_{3} \operatorname{Tr} \sigma^{(1)}+2 \pi \mathrm{i} \tau_{2} \operatorname{Tr} \sigma^{(2)} \\
& -2 \pi \mathrm{i}\left(\tau_{1}+\tau_{2}\right) \operatorname{Tr} \sigma^{(3)} .
\end{aligned}
$$

Here we have again renamed indices in such a way that the labelling of the $\sigma$-variables follows the same order in which the gauge nodes are drawn in Fig. 9.

Next, we determine the classical vacuum for the quivers in this duality chain by equating the classical twisted chiral superpotentials for each dual pairs. In Table 5 we report the results for the three new quivers $\widehat{Q}_{1}, Q_{3}$ and $\widehat{Q}_{5}$ of this sequence.

Using this information and following the same procedure described above, we can find the $q$ vs $\Lambda$ map and the contour prescription that has to be used in the localization formula in order to match term-by-term the superpotential with the one obtained from solving the twisted chiral ring equations.

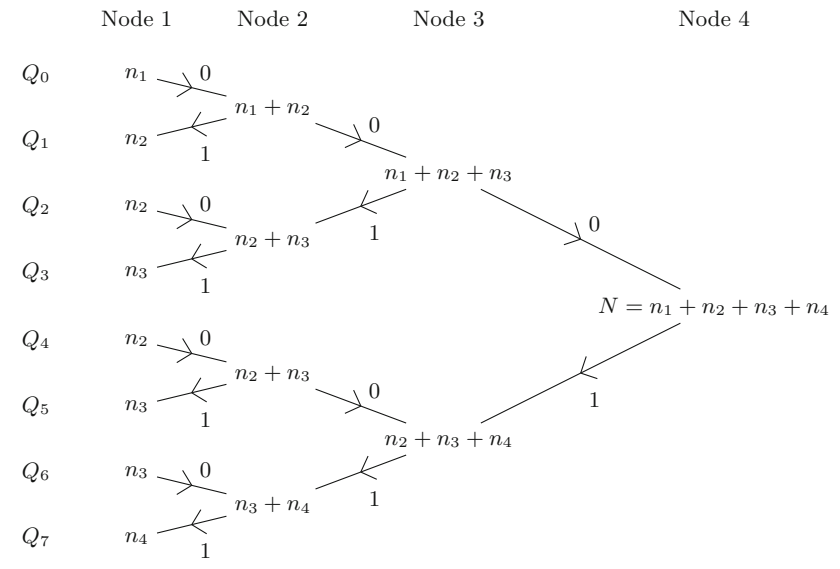

Fig. 10 The linear quivers that are Seiberg-dual to the oriented quiver $Q_{0}$. To each link we associate 0 or 1 depending whether it is rightward or leftward

Of course, we do not repeat the derivation of these results since the calculations are a straightforward generalization of what we did for the other duality chain, and we simply collect our findings for the three new quivers $\widehat{Q}_{1}, Q_{3}$ and $\widehat{Q}_{5}$ in Table 6 . We have checked the validity of our proposal up to two instantons, while some details on the results at the one-instanton level can be found in Appendix B.

\section{Proposal for generic linear quivers}

The detailed analysis of the previous section shows that in the 4-node case there are eight linear quivers related to each other by duality: the seven ones found in the sequence of Fig. 8, and the quiver $Q_{3}$ in the sequence of Fig. 9. If we consider these eight linear quivers all together, a nice structure emerges as illustrated in Fig. 10 where we exhibit the ranks of the nodes of the various quivers and their connections.

We recall that the ranks of the nodes of the initial oriented quiver $Q_{0}$ can be obtained from the vector $\boldsymbol{n}=$ $\left(n_{1}, n_{2}, n_{3}, n_{4}\right)$ as discussed in Sect. 2 (see (3)). Then, given the action of Seiberg duality, it is easy to realize that the ranks
Table 6 For the quivers $\widehat{Q}_{1}, Q_{3}$ and $\widehat{Q}_{5}$ drawn in Fig. 9, we list the relations (up to signs) between the ramified instanton counting parameters

\begin{tabular}{|c|c|c|c|c|}
\hline Quiver & $q_{1}$ & $q_{2}$ & $q_{3}$ & JK vector \\
\hline$\widehat{Q}_{1}$ & $\left(\Lambda_{1}^{\widehat{Q}_{1}}\right)^{n_{1}+n_{2}}$ & $\left(\Lambda_{2}^{\widehat{Q}_{1}}\right)^{n_{2}+n_{3}}$ & $\frac{\left(\Lambda_{3}^{\widehat{Q}_{1}}\right)^{n_{2}+2 n_{3}+n_{4}}}{\left(\Lambda_{2}^{\hat{Q}_{1}}\right)^{n_{2}+n_{3}}}$ & $-\zeta_{1}^{\widehat{Q}_{1}} \chi_{1}-\zeta_{2}^{\widehat{Q}_{1}} \chi_{2}-\zeta_{3}^{\widehat{Q}_{1}} \chi_{3}+\zeta_{4} \chi_{4}$ \\
\hline$Q_{3}$ & $\left(\Lambda_{2}^{Q_{3}}\right)^{n_{1}+n_{2}}$ & $\left(\Lambda_{1}^{Q_{3}}\right)^{n_{2}+n_{3}}$ & $\frac{\left(\Lambda_{3}^{Q_{3}}\right)^{N+n_{2}+n_{3}}}{\left(\Lambda_{1}^{Q_{3}}\right)^{n_{2}+n_{3}}\left(\Lambda_{2}^{Q_{3}}\right)^{n_{1}+n_{2}}}$ & $-\zeta_{1}^{Q_{3}} \chi_{2}-\zeta_{2}^{Q_{3}} \chi_{1}-\zeta_{3}^{Q_{3}} \chi_{3}+\zeta_{4} \chi_{4}$ \\
\hline$\widehat{Q}_{5}$ & $\frac{\left(\Lambda_{3}^{\widehat{Q}_{5}}\right)^{n_{1}+2 n_{2}+n_{3}}}{\left(\Lambda_{2}^{\hat{Q}_{5}}\right)^{n_{2}+n_{3}}}$ & $\left(\Lambda_{2}^{\widehat{Q}_{5}}\right)^{n_{2}+n_{3}}$ & $\left(\Lambda_{1}^{\widehat{Q}_{5}}\right)^{n_{3}+n_{4}}$ & $-\zeta_{1}^{\widehat{Q}_{5}} \chi_{3}-\zeta_{2}^{\widehat{Q}_{5}} \chi_{2}-\zeta_{3}^{\widehat{Q}_{5}} \chi_{1}-\zeta_{4} \chi_{4}$ \\
\hline
\end{tabular}
$q_{I}$ and the strong coupling scales $\Lambda_{I}$, and also the $\mathrm{JK}$ reference vector that selects the contour prescription needed to compute the ramified instanton partition function using the localization formula 
of the nodes of the other quivers can be obtained from vectors that are a permutation of the entries of $\boldsymbol{n}$. For example, for the quiver $Q_{2}$ the ranks can be obtained from $\left(n_{2}, n_{3}, n_{1}, n_{4}\right)$, while for quiver $Q_{6}$ they are obtained from $\left(n_{3}, n_{4}, n_{2}, n_{1}\right)$. It is not difficult to realize that all these permuted vectors can be written as

$P_{2}^{s_{3}} P_{3}^{s_{2}} P_{4}^{s_{1}} \boldsymbol{n}$,

where $s_{i}=0,1$ and $P_{k}$ is the cyclic permutation on the first $k$ elements out of 4 . In matrix form, we have

$$
\begin{aligned}
& P_{2}=\left(\begin{array}{llll}
0 & 1 & 0 & 0 \\
1 & 0 & 0 & 0 \\
0 & 0 & 1 & 0 \\
0 & 0 & 0 & 1
\end{array}\right), \\
& P_{3}=\left(\begin{array}{llll}
0 & 1 & 0 & 0 \\
0 & 0 & 1 & 0 \\
1 & 0 & 0 & 0 \\
0 & 0 & 0 & 1
\end{array}\right), \\
& P_{4}=\left(\begin{array}{llll}
0 & 1 & 0 & 0 \\
0 & 0 & 1 & 0 \\
0 & 0 & 0 & 1 \\
1 & 0 & 0 & 0
\end{array}\right) .
\end{aligned}
$$

We therefore see that each linear quiver $Q_{i}$ can be labelled by the set $\mathbf{s}=\left(s_{1}, s_{2}, s_{3}\right)$ identifying the permutation

$$
P[\mathbf{s}]=P_{2}^{s_{3}} P_{3}^{s_{2}} P_{4}^{s_{1}}
$$

which determines the ranks of the various nodes. For example, the quiver $Q_{3}$ corresponds to $(0,1,1)$ and the quiver $Q_{5}$ to $(1,0,1)$. For any quiver, its corresponding $\mathbf{S}$ can be easily read from Fig. 10 by looking at the labels 0 and 1 on the links connecting the nodes, starting from the rightmost one and moving leftwards. Notice that, with the conventions we have chosen, the quiver $Q_{i}$ turns out to be labelled by the vector $\mathbf{s}$ that represents the number $i$ written in binary notation.

The permutation $P[\mathbf{s}]$ can be represented in an irreducible way in terms of $3 \times 3$ matrices as follows

$$
\widehat{P}[\mathbf{s}]=\widehat{P}_{2}^{s_{3}} \widehat{P}_{3}^{s_{2}} \widehat{P}_{4}^{s_{1}}
$$

where

$$
\begin{aligned}
& \widehat{P}_{2}=\left(\begin{array}{ccc}
-1 & 0 & 0 \\
1 & 1 & 0 \\
0 & 0 & 1
\end{array}\right), \\
& \widehat{P}_{3}=\left(\begin{array}{ccc}
0 & 1 & 0 \\
-1 & -1 & 0 \\
1 & 1 & 1
\end{array}\right), \\
& \widehat{P}_{4}=\left(\begin{array}{ccc}
0 & 1 & 0 \\
0 & 0 & 1 \\
-1 & -1 & -1
\end{array}\right) .
\end{aligned}
$$

This defines the action on the FI couplings. Indeed, if we introduce the vector $\zeta=\left(\zeta_{1}, \zeta_{2}, \zeta_{3}\right)$ with the FI parameters of the first quiver $Q_{0}$, then it is easy to check that

$\left(\zeta_{1}^{Q_{i}}, \zeta_{2}^{Q_{i}}, \zeta_{3}^{Q_{i}}\right)=\widehat{P}[\mathbf{s}] \zeta$

For example, for $Q_{4}$ we have

$\widehat{P}[(1,0,0)] \zeta=\widehat{P}_{4} \zeta=\left(\zeta_{2}, \zeta_{3},-\zeta_{1}-\zeta_{2}-\zeta_{3}\right)$,

which indeed are the FI parameters of $Q_{4}$, as one can see from the superpotential $\mathcal{W}_{\mathrm{cl}}^{Q_{4}}$ in (43).

This formalism can be nicely used also to describe how the variables $\chi_{I}$ appearing in the localization integrals are associated to the various nodes of the quiver. From the detailed analysis of Sect. 4 , we see that $\chi_{4}$ is always associated to the last $4 \mathrm{~d}$ node of the quiver, while the other three variables $\chi_{1}, \chi_{2}$ and $\chi_{3}$ are associated to the first three $2 \mathrm{~d}$ nodes in a permutation determined by the $q$ vs $\Lambda$ map. Moreover, we see that two quivers whose vectors $\mathbf{s}$ only differ by the value of $s_{3}$ have the same permutation and that this permutation involves only cyclic rearrangements of the first two or the first three variables described by $P_{2}$ and $P_{3}$. In particular, introducing the vector $\chi=\left(\chi_{1}, \chi_{2}, \chi_{3}, \chi_{4}\right)$, we can check that

$\chi[\mathbf{s}]=P_{2}^{s_{2}} P_{3}^{s_{1}} \chi$

correctly describes the correspondence between the nodes of the quiver and the $\chi$-variables. For example, for $Q_{6}$ we find that $\chi[(1,1,0)]=P_{2} P_{3} \chi=\left(\chi_{3}, \chi_{2}, \chi_{1}, \chi_{4}\right)$, which is indeed the correct sequence of $\chi$-variables for $Q_{6}$ as one can see from Table 3 .

We are now in the position of using this formalism to write the JK reference vector for any linear quiver in a compact form. To this aim, we first extend the three-component vector (82) by adding to it a fourth component according to

$$
\zeta[\mathbf{s}]=\left(\widehat{P}[\mathbf{s}] \zeta, \pm \zeta_{4}\right)=\left(\zeta_{1}^{Q_{i}}, \zeta_{2}^{Q_{i}}, \zeta_{3}^{Q_{i}}, \pm \zeta_{4}\right)
$$

Here $\zeta_{4}$ is a positive parameter that is always bigger than $\left|\zeta_{I}^{Q_{i}}\right|$ for $I=1,2,3$. The sign in (85) depends whether the $4 \mathrm{~d}$ node of the quiver provides fundamental $(+)$ or antifundamental $(-)$ matter to the last $2 \mathrm{~d}$ node. By considering the detailed structure of the various quivers, we see that in the first four quivers from $Q_{0}$ to $Q_{3}$ the 4 d node provide antifundamental matter, while in the last four ones from $Q_{4}$ to $Q_{7}$ it provides fundamental flavors. This means that the sign in (85) can also be written as $(-1)^{s_{1}+1}$. With these positions, it is easy to realize that the $\mathrm{JK}$ vectors described in the previous section can all be compactly written as follows:

$\eta_{Q_{i}}=-\zeta[\mathbf{s}] \cdot \chi[\mathbf{s}]$

This analysis can be extended to linear quivers with $M$ nodes in a straightforward manner. In this case we have $(M-$ 
Table 7 The dictionary between the various features of surface operators in the two descriptions, as monodromy defects and as coupled $2 \mathrm{~d} / 4 \mathrm{~d}$ quivers

\begin{tabular}{ll}
\hline Monodromy defect & $2 \mathrm{~d} / 4 \mathrm{~d}$ quiver models \\
\hline Partition of $N:\left(n_{1}, n_{2}, \ldots, n_{M}\right)$ & Ranks of $2 \mathrm{~d}$ gauge nodes \\
4d Coulomb vev's & $2 \mathrm{~d}$ twisted masses \\
Partition of Coulomb vev's & Classical (massive) vacuum \\
Ramified instanton & $2 \mathrm{~d} / 4 \mathrm{~d}$ strong coupling scales \\
$\quad$ counting parameters & \\
$q_{I}, q_{M}$ & $\Lambda_{I}, \Lambda_{4 \mathrm{~d}}$ \\
$\mathcal{W}_{\text {inst }}(a, q)$ & $\left.\mathcal{W}\left(\sigma, a, \Lambda_{I}, \Lambda_{4 \mathrm{~d}}\right)\right|_{\sigma_{\star}}$ \\
Contour prescription & $2 \mathrm{~d}$ Seiberg duality frame \\
\hline
\end{tabular}

1) binary choices corresponding to $2^{M-1}$ linear quivers that are related to each other by Seiberg duality. Therefore, they can be labelled by a vector $\mathbf{s}=\left(s_{1}, s_{2}, \ldots, s_{M-1}\right)$ with $s_{i}=$ 0,1 . For each choice, the ranks of the $M$ nodes are determined by the permutation

$$
P[\mathbf{s}]=P_{2}^{s_{M-1}} P_{3}^{s_{M-2}} \cdots P_{M-1}^{s_{2}} P_{M}^{s_{1}}
$$

where $P_{k}$ permutes the first $k$ numbers, while the FI parameters of the $(M-1)$ nodes are obtained using $\widehat{P}[\mathbf{s}]$, which represents the permutation $P[\mathbf{s}]$ in an irreducible way in an ( $M-1)$-dimensional space. Generalizing (85) to the $M$-node case in an obvious way, and defining

$\chi[\mathbf{s}]=P_{2}^{s_{M-2}} P_{3}^{s_{M-3}} \cdots P_{M-2}^{s_{2}} P_{M-1}^{s_{1}} \chi$

where $\chi=\left(\chi_{1}, \ldots, \chi_{M}\right)$, it is natural to propose that the JK reference vector for a generic quiver $Q_{i}$ is

$\eta_{Q_{i}}=-\zeta[\mathbf{s}] \cdot \chi[\mathbf{s}]=-\sum_{I=1}^{M-1} \zeta_{I}^{Q_{i}} \chi_{\alpha(I)} \pm \zeta_{4} \chi_{4}$

where $\alpha(I)$ is determined by the permutation in (88). We have verified in several examples the validity of this proposal.

\section{Summary of results}

In this paper we have discussed in detail the relation between two distinct realizations of surface operators: as monodromy defects and as coupled $2 \mathrm{~d} / 4 \mathrm{~d}$ quiver gauge theories. The main features of these two points of view and their relations are summarized in Table 7.

Establishing a precise correspondence between different integration contour prescriptions in the ramified instanton partition function for a monodromy defect and different quiver theories related to each other by a Seiberg duality has been the main focus of our present work. Dual quivers have different ultraviolet realizations but share the same infrared physics and thus the (massive) vacua of their low-energy the- ories can be mapped onto each other. These massive vacua are obtained by extremizing the effective twisted chiral superpotential of the $2 \mathrm{~d} / 4 \mathrm{~d}$ quiver. The evaluation of the effective superpotential in a particular vacuum is in turn mapped to the twisted superpotential which is extracted from the ramified instanton partition function with a specific contour of integration.

For surface operators in pure $\mathcal{N}=2$ gauge theories, like the ones we have considered in this paper, residue theorem ensures that one always obtains the same superpotential irrespective of the contour of integration chosen. Nevertheless, by a careful study of the individual residues that contribute to the superpotential, we have been able to map distinct contours on the localization side to distinct Seiberg-dual 2d quivers coupled to the same $4 \mathrm{~d} \mathrm{SU}(N)$ flavour group. The duality frame one chooses affects the details of the other entries in the table above, such as the choice of the classical vacuum and the map between the ramified instanton counting parameters $q_{I}$ and the strong coupling scales $\Lambda_{I}$. We initially restricted ourselves to systems with four nodes to exhibit our explicit results, but in the end we have generalized our analysis to linear quivers with an arbitrary number of nodes providing the map between the data of the quiver and the corresponding JK prescription, which takes a universal form.

There is one caveat to our analysis. All quivers we have studied so far, have only a single $2 \mathrm{~d}$ node that is connected to the flavour node that is gauged in $4 \mathrm{~d}$. It is only for such cases that the coupling of the $2 \mathrm{~d}$ degrees of freedom to the $4 \mathrm{~d}$ theory via its resolvent gives results that are consistent with those obtained using localization methods in the monodromy defect approach. It would be very interesting to understand whether quivers with more $2 \mathrm{~d}$ nodes connected to the $4 \mathrm{~d}$ node also have an interpretation as surface operators in a $4 \mathrm{~d}$ gauge theory. Furthermore, there are many worthwhile but yet unexplored directions to pursue, such as the extension of our analysis to (conformal) SQCD models for which the integrands of ramified instanton partition function may have non-vanishing residues at infinity, or the lift of our techniques to five dimensions to study surface operators from the point of view of $3 \mathrm{~d} / 5 \mathrm{~d}$ coupledsystems, with possible Chern-Simons interactions. We leave these extensions and generalizations to future work.

Acknowledgements We would like to thank Stefano Cremonesi and Amihay Hanany for many useful discussions. S.K.A. would especially like to thank the Physics Department of the University of Torino and the Torino Section of INFN for hospitality during the final stages of this work. M.B, M.F., R.R.J. and A.L. would like to thank the "Galileo Galilei Institute for Theoretical Physics" in Florence for hospitality. The work of M.B., M.F., R.R.J. and A.L. is partially supported by the MIUR PRIN Contract 2015MP2CX4 "Non-perturbative aspects of gauge theories and strings". The work of A.L. is partially supported by the "Fondi Ricerca Locale dell'Università del Piemonte Orientale". 
Data Availability Statement This manuscript has no associated data or the data will not be deposited. [Authors' comment: There are no data associated with this manuscript.]

Open Access This article is distributed under the terms of the Creative Commons Attribution 4.0 International License (http://creativecomm ons.org/licenses/by/4.0/), which permits unrestricted use, distribution, and reproduction in any medium, provided you give appropriate credit to the original author(s) and the source, provide a link to the Creative Commons license, and indicate if changes were made.

Funded by SCOAP ${ }^{3}$.

\section{A Localization results at one-instanton level}

In this Appendix we collect the localization results at the one-instanton level for the different contours of integrations, corresponding to the different quivers discussed in Sects. 4 and 4.3. The twisted superpotential extracted from the partition function (8) is expressed as a sum of residues, and at the one-instanton level it can be easily derived from (31).

Integration contour $\left(\left.\chi_{1}\right|_{+},\left.\chi_{2}\right|_{+},\left.\chi_{3}\right|_{+},\left.\chi_{4}\right|_{-}\right)$:

$$
\begin{aligned}
\mathcal{W}_{1-\text { inst }}= & \sum_{s \in \mathcal{N}_{1}} \frac{(-1)^{n_{1}} q_{1}}{\prod_{r \in \widehat{\mathcal{N}}_{1} \cup \mathcal{N}_{2}}\left(a_{s}-a_{r}\right)} \\
& +\sum_{t \in \mathcal{N}_{2}} \frac{(-1)^{n_{2}} q_{2}}{\prod_{r \in \widehat{\mathcal{N}}_{2} \cup \mathcal{N}_{3}}\left(a_{t}-a_{r}\right)} \\
& +\sum_{u \in \mathcal{N}_{3}} \frac{(-1)^{n_{3}} q_{3}}{\prod_{r \in \widehat{\mathcal{N}}_{3} \cup \mathcal{N}_{4}}\left(a_{u}-a_{r}\right)} \\
& +\sum_{s \in \mathcal{N}_{1}} \frac{(-1)^{n_{4}+1} q_{4}}{\prod_{r \in \mathcal{N}_{4} \cup \widehat{\mathcal{N}}_{1}}\left(a_{s}-a_{r}\right)} .
\end{aligned}
$$

Integration contour $\left(\left.\chi_{1}\right|_{-},\left.\chi_{2}\right|_{+},\left.\chi_{3}\right|_{+},\left.\chi_{4}\right|_{-}\right)$:

$$
\begin{aligned}
\mathcal{W}_{1-\text { inst }}= & \sum_{t \in \mathcal{N}_{2}} \frac{(-1)^{n_{1}+1} q_{1}}{\prod_{r \in \mathcal{N}_{1} \cup \widehat{\mathcal{N}}_{2}}\left(a_{t}-a_{r}\right)} \\
& +\sum_{t \in \mathcal{N}_{2}} \frac{(-1)^{n_{2}} q_{2}}{\prod_{r \in \widehat{\mathcal{N}_{2}} \cup \mathcal{N}_{3}}\left(a_{t}-a_{r}\right)} \\
& +\sum_{u \in \mathcal{N}_{3}} \frac{(-1)^{n_{3}} q_{3}}{\prod_{r \in \widehat{\mathcal{N}}_{3} \cup \mathcal{N}_{4}}\left(a_{u}-a_{r}\right)} \\
& +\sum_{s \in \mathcal{N}_{1}} \frac{(-1)^{n_{4}+1} q_{4}}{\prod_{r \in \mathcal{N}_{4} \cup \widehat{\mathcal{N}}_{1}}\left(a_{s}-a_{r}\right)} .
\end{aligned}
$$

Integration contour $\left(\left.\chi_{2}\right|_{+},\left.\chi_{1}\right|_{-},\left.\chi_{3}\right|_{+},\left.\chi_{4}\right|_{-}\right)$:

The one-instanton superpotential for this integration contour is the same as in (91) since at this order there is no difference between the two cases.
Integration contour $\left(\left.\chi_{2}\right|_{+},\left.\chi_{3}\right|_{+},\left.\chi_{1}\right|_{-},\left.\chi_{4}\right|_{+}\right)$:

$$
\begin{aligned}
\mathcal{W}_{1-\text { inst }}= & \sum_{t \in \mathcal{N}_{2}} \frac{(-1)^{n_{1}+1} q_{1}}{\prod_{r \in \mathcal{N}_{1} \cup \widehat{\mathcal{N}}_{2}}\left(a_{t}-a_{r}\right)} \\
& +\sum_{t \in \mathcal{N}_{2}} \frac{(-1)^{n_{2}} q_{2}}{\prod_{r \in \widehat{\mathcal{N}}_{2} \cup \mathcal{N}_{3}}\left(a_{t}-a_{r}\right)} \\
& +\sum_{u \in \mathcal{N}_{3}} \frac{(-1)^{n_{3}} q_{3}}{\prod_{r \in \widehat{\mathcal{N}}_{3} \cup \mathcal{N}_{4}}\left(a_{u}-a_{r}\right)} \\
& +\sum_{v \in \mathcal{N}_{4}} \frac{(-1)^{n_{4}} q_{4}}{\prod_{r \in \widehat{\mathcal{N}}_{4} \cup \mathcal{N}_{1}}\left(a_{v}-a_{r}\right)} .
\end{aligned}
$$

Integration contour $\left(\left.\chi_{2}\right|_{-},\left.\chi_{3}\right|_{+},\left.\chi_{1}\right|_{-},\left.\chi_{4}\right|_{+}\right)$:

$$
\begin{aligned}
\mathcal{W}_{1-\text { inst }}= & \sum_{t \in \mathcal{N}_{2}} \frac{(-1)^{n_{1}+1} q_{1}}{\prod_{r \in \mathcal{N}_{1} \cup \widehat{\mathcal{N}}_{2}}\left(a_{t}-a_{r}\right)} \\
& +\sum_{u \in \mathcal{N}_{3}} \frac{(-1)^{n_{2}+1} q_{2}}{\prod_{r \in \mathcal{N}_{2} \cup \widehat{\mathcal{N}}_{3}}\left(a_{t}-a_{r}\right)} \\
& +\sum_{u \in \mathcal{N}_{3}} \frac{(-1)^{n_{3}} q_{3}}{\prod_{r \in \widehat{\mathcal{N}}_{3} \cup \mathcal{N}_{4}}\left(a_{u}-a_{r}\right)} \\
& +\sum_{v \in \mathcal{N}_{4}} \frac{(-1)^{n_{4}} q_{4}}{\prod_{r \in \widehat{\mathcal{N}}_{4} \cup \mathcal{N}_{1}}\left(a_{v}-a_{r}\right)}
\end{aligned}
$$

Integration contour $\left(\left.\chi_{3}\right|_{+},\left.\chi_{2}\right|_{-},\left.\chi_{1}\right|_{-},\left.\chi_{4}\right|_{+}\right)$:

The one-instanton superpotential for this integration contour is the same as in (93) since at this order there is no difference between the two cases.

Integration contour $\left(\left.\chi_{3}\right|_{-},\left.\chi_{2}\right|_{-},\left.\chi_{1}\right|_{-},\left.\chi_{4}\right|_{+}\right)$:

$$
\begin{aligned}
\mathcal{W}_{1-\text { inst }}= & \sum_{t \in \mathcal{N}_{2}} \frac{(-1)^{n_{1}+1} q_{1}}{\prod_{r \in \mathcal{N}_{1} \cup \widehat{\mathcal{N}}_{2}}\left(a_{t}-a_{r}\right)} \\
& +\sum_{u \in \mathcal{N}_{3}} \frac{(-1)^{n_{2}+1} q_{2}}{\prod_{r \in \mathcal{N}_{2} \cup \widehat{\mathcal{N}}_{3}}\left(a_{u}-a_{r}\right)} \\
& +\sum_{v \in \mathcal{N}_{4}} \frac{(-1)^{n_{3}+1} q_{3}}{\prod_{r \in \mathcal{N}_{3} \cup \widehat{\mathcal{N}}_{4}}\left(a_{v}-a_{r}\right)} \\
& +\sum_{v \in \mathcal{N}_{4}} \frac{(-1)^{n_{4}} q_{4}}{\prod_{r \in \widehat{\mathcal{N}}_{4} \cup \mathcal{N}_{1}}\left(a_{v}-a_{r}\right)} .
\end{aligned}
$$


Integration contour $\left(\left.\chi_{1}\right|_{+},\left.\chi_{2}\right|_{-},\left.\chi_{3}\right|_{+},\left.\chi_{4}\right|_{-}\right)$:

$$
\begin{aligned}
\mathcal{W}_{1-\text { inst }}= & \sum_{s \in \mathcal{N}_{1}} \frac{(-1)^{n_{1}} q_{1}}{\prod_{r \in \widehat{\mathcal{N}}_{1} \cup \mathcal{N}_{2}}\left(a_{s}-a_{r}\right)} \\
& +\sum_{u \in \mathcal{N}_{3}} \frac{(-1)^{n_{2}+1} q_{2}}{\prod_{r \in \mathcal{N}_{2} \cup \widehat{\mathcal{N}}_{3}}\left(a_{u}-a_{r}\right)} \\
& +\sum_{u \in \mathcal{N}_{3}} \frac{(-1)^{n_{3}} q_{3}}{\prod_{r \in \widehat{\mathcal{N}}_{3} \cup \mathcal{N}_{4}}\left(a_{u}-a_{r}\right)} \\
& +\sum_{s \in \mathcal{N}_{1}} \frac{(-1)^{n_{4}+1} q_{4}}{\prod_{r \in \mathcal{N}_{4} \cup \widehat{\mathcal{N}}_{1}}\left(a_{s}-a_{r}\right)} .
\end{aligned}
$$

Integration contour $\left(\left.\chi_{2}\right|_{-},\left.\chi_{1}\right|_{-},\left.\chi_{3}\right|_{+},\left.\chi_{4}\right|_{-}\right)$:

$$
\begin{aligned}
\mathcal{W}_{1-\text { inst }}= & \sum_{t \in \mathcal{N}_{2}} \frac{(-1)^{n_{1}+1} q_{1}}{\prod_{r \in \mathcal{N}_{1} \cup \widehat{\mathcal{N}}_{2}}\left(a_{t}-a_{r}\right)} \\
& +\sum_{u \in \mathcal{N}_{3}} \frac{(-1)^{n_{2}+1} q_{2}}{\prod_{r \in \mathcal{N}_{2} \cup \widehat{\mathcal{N}}_{3}}\left(a_{u}-a_{r}\right)} \\
& +\sum_{u \in \mathcal{N}_{3}} \frac{(-1)^{n_{3}} q_{3}}{\prod_{r \in \widehat{\mathcal{N}_{3}} \cup \mathcal{N}_{4}}\left(a_{u}-a_{r}\right)} \\
& +\sum_{s \in \mathcal{N}_{1}} \frac{(-1)^{n_{4}+1} q_{4}}{\prod_{r \in \mathcal{N}_{4} \cup \widehat{\mathcal{N}}_{1}}\left(a_{s}-a_{r}\right)} .
\end{aligned}
$$

Integration contour $\left(\left.\chi_{3}\right|_{-},\left.\chi_{2}\right|_{+},\left.\chi_{1}\right|_{-},\left.\chi_{4}\right|_{+}\right)$:

$$
\begin{aligned}
\mathcal{W}_{1-\text { inst }}= & \sum_{t \in \mathcal{N}_{2}} \frac{(-1)^{n_{1}+1} q_{1}}{\prod_{r \in \mathcal{N}_{1} \cup \widehat{\mathcal{N}}_{2}}\left(a_{t}-a_{r}\right)} \\
& +\sum_{t \in \mathcal{N}_{2}} \frac{(-1)^{n_{2}} q_{2}}{\prod_{r \in \widehat{\mathcal{N}}_{2} \cup \mathcal{N}_{3}}\left(a_{t}-a_{r}\right)} \\
& +\sum_{v \in \mathcal{N}_{4}} \frac{(-1)^{n_{3}+1} q_{3}}{\prod_{r \in \mathcal{N}_{3} \cup \widehat{\mathcal{N}}_{4}}\left(a_{v}-a_{r}\right)} \\
& +\sum_{u \in \mathcal{N}_{4}} \frac{(-1)^{n_{4}} q_{4}}{\prod_{r \in \widehat{\mathcal{N}}_{4} \cup \mathcal{N}_{1}}\left(a_{u}-a_{r}\right)}
\end{aligned}
$$

\section{B Chiral ring equations and superpotentials at the one-instanton level}

\section{Quiver $Q_{0}$}

We begin by considering the first quiver $Q_{0}$ of the two duality chains of Figs. 8 and 9, namely

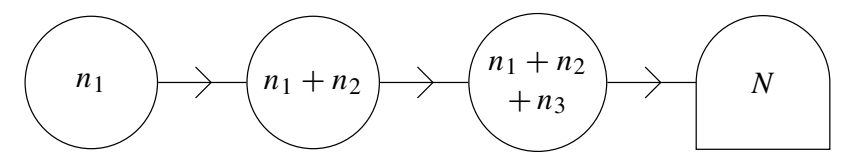

The corresponding chiral ring equations have already been written in Sect. 2.3, but we rewrite them here for convenience

$$
\begin{aligned}
\mathcal{Q}_{2}\left(\sigma_{s}^{(1)}\right)= & \Lambda_{1}^{n_{1}+n_{2}} \\
\mathcal{Q}_{3}\left(\sigma_{t}^{(2)}\right)= & (-1)^{n_{1}} \Lambda_{2}^{n_{2}+n_{3}} \mathcal{Q}_{1}\left(\sigma_{t}^{(2)}\right) \\
\mathcal{P}_{N}\left(\sigma_{u}^{(3)}\right)= & (-1)^{n_{1}+n_{2}}\left(\Lambda_{3}^{n_{3}+n_{4}} \mathcal{Q}_{2}\left(\sigma_{u}^{(3)}\right)\right. \\
& \left.+\frac{\Lambda_{4 \mathrm{~d}}^{2 N}}{\Lambda_{3}^{n_{3}+n_{4}} \mathcal{Q}_{2}\left(\sigma_{u}^{(3)}\right)}\right)
\end{aligned}
$$

for $s \in \mathcal{N}_{1}, t \in \mathcal{N}_{1} \cup \mathcal{N}_{2}$, and $u \in \mathcal{N}_{1} \cup \mathcal{N}_{2} \cup \mathcal{N}_{3}$, respectively.

We look for solutions of these equations that are of the form $\sigma_{\star}^{(I)}=\sigma_{\mathrm{cl}}^{(I)}+\delta \sigma^{(I)}$ with the classical vacuum given in the first row of Table 1 . We work at the lowest order in the quantum fluctuations, proportional to $9 \Lambda_{1}^{n_{1}+n_{2}}, \Lambda_{2}^{n_{2}+n_{3}}$, $\Lambda_{3}^{n_{3}+n_{4}}$ and $\Lambda_{4 \mathrm{~d}}^{2 N} /\left(\Lambda_{1}^{n_{1}+n_{2}} \Lambda_{2}^{n_{2}+n_{3}} \Lambda_{3}^{n_{3}+n_{4}}\right)$. With this Ansatz, equation (98a) gives

$\delta \sigma_{s}^{(1)}-\delta \sigma_{s}^{(2)}=\frac{\Lambda_{1}^{n_{1}+n_{2}}}{\prod_{r \in \widehat{\mathcal{N}_{1}} \cup \mathcal{N}_{2}}\left(a_{s}-a_{r}\right)}$

for $s \in \mathcal{N}_{1}$, while equation (98b) yields

$\delta \sigma_{t}^{(2)}-\delta \sigma_{t}^{(3)}=\frac{(-1)^{n_{1}+1} \Lambda_{1}^{n_{1}+n_{2}} \Lambda_{2}^{n_{2}+n_{3}}}{\prod_{u \in \widehat{\mathcal{N}}_{1} \cup \mathcal{N}_{2} \cup \mathcal{N}_{3}}\left(a_{t}-a_{u}\right) \prod_{r \in \mathcal{N}_{2}}\left(a_{t}-a_{r}\right)}$

when $t \in \mathcal{N}_{1}$, and

$\delta \sigma_{t}^{(2)}-\delta \sigma_{t}^{(3)}=\frac{(-1)^{n_{1}} \Lambda_{2}^{n_{2}+n_{3}}}{\prod_{r \in \widehat{\mathcal{N}}_{2} \cup \mathcal{N}_{3}}\left(a_{t}-a_{r}\right)}$

when $t \in \mathcal{N}_{2}$. The right hand side of (100) appears as a higher-order term and hence one could naively think that it may be discarded. However, one should not do that, since it contributes to the lowest-order term in (98c).

Let us now consider (98c). First of all, we observe that, since we are at the lowest order in the ramified instanton expansion, the quantum polynomial $\mathcal{P}_{N}(z)$ can be replaced with its classical counterpart

$\mathcal{P}_{N}(z)=\prod_{i=1}^{N}\left(z-a_{i}\right)$.

Then, we proceed to solve (98c) block by block. In the first block when $u \in \mathcal{N}_{1}$ and $\mathcal{Q}_{2}\left(\sigma_{u}^{(3)}\right)$ has a zero, it is the term

\footnotetext{
9 According to Table 2 and Eq. (57) this corresponds to the lowest order in the $q_{I}$ parameters.
} 
proportional to $\Lambda_{4 \mathrm{~d}}^{2 N}$ in the right hand side of (98c) that contributes to lowest order, and we have

$$
\begin{gathered}
\prod_{r \in \widehat{\mathcal{N}}_{1} \cup \mathcal{N}_{2} \cup \mathcal{N}_{3} \cup \mathcal{N}_{4}}\left(a_{u}-a_{r}\right) \delta \sigma_{u}^{(3)} \\
=\frac{(-1)^{n_{1}+n_{2}} \Lambda_{4 \mathrm{~d}}^{2 N}}{\Lambda_{3}^{n_{3}+n_{4}} \prod_{r \in \widehat{\mathcal{N}}_{1} \cup \mathcal{N}_{2}}\left(a_{u}-a_{r}\right)\left(\delta \sigma_{u}^{(3)}-\delta \sigma_{u}^{(2)}\right)} .
\end{gathered}
$$

Inserting (100), we find

$$
\delta \sigma_{u}^{(3)}=\frac{(-1)^{n_{2}} \Lambda_{4 \mathrm{~d}}^{2 N}}{\Lambda_{1}^{n_{1}+n_{2}} \Lambda_{2}^{n_{2}+n_{3}} \Lambda_{3}^{n_{3}+n_{4}} \prod_{r \in \widehat{\mathcal{N}}_{1} \cup \mathcal{N}_{4}}\left(a_{u}-a_{r}\right)} .
$$

In the second block when $u \in \mathcal{N}_{2}$ and $\mathcal{Q}_{2}\left(\sigma_{u}^{(3)}\right)$ has a zero, it is again the term proportional to $\Lambda_{4 \mathrm{~d}}^{2 N}$ in the right hand side of (98c) that can contribute to the solution at the lowest order. Indeed, we have

$$
\begin{aligned}
& \prod_{r \in \mathcal{N}_{1} \cup \widehat{\mathcal{N}}_{2} \cup \mathcal{N}_{3} \cup \mathcal{N}_{4}}\left(a_{u}-a_{r}\right) \delta \sigma_{u}^{(3)} \\
= & \frac{(-1)^{n_{1}+n_{2}} \Lambda_{4 \mathrm{~d}}^{2 N}}{\Lambda_{3}^{n_{3}+n_{4}} \prod_{r \in \mathcal{N}_{1} \cup \widehat{\mathcal{N}}_{2}}\left(a_{u}-a_{r}\right)\left(\delta \sigma_{u}^{(3)}-\delta \sigma_{u}^{(2)}\right)} .
\end{aligned}
$$

Substituting (101), we get

$$
\begin{aligned}
\delta \sigma_{u}^{(3)}= & \frac{(-1)^{n_{2}+1} \Lambda_{4 \mathrm{~d}}^{2 N}}{\Lambda_{2}^{n_{2}+n_{3}} \Lambda_{3}^{n_{3}+n_{4}} \prod_{r \in \mathcal{N}_{1} \cup \widehat{\mathcal{N}}_{2} \cup \mathcal{N}_{4}}\left(a_{u}-a_{r}\right)} \\
& \times \frac{1}{\prod_{s \in \mathcal{N}_{1}}\left(a_{u}-a_{s}\right)} .
\end{aligned}
$$

This term, however, is of higher order and thus can be neglected at the one-instanton level.

Finally, in the third block when $u \in \mathcal{N}_{3}$ and $\mathcal{Q}_{2}\left(\sigma_{u}^{(3)}\right)$ has no zeroes, it is the term proportional to $\Lambda_{3}^{n_{3}+n_{4}}$ in the right hand side of (98c) that contributes. Indeed, we find

$\delta \sigma_{u}^{(3)}=\frac{(-1)^{n_{1}+n_{2}} \Lambda_{3}^{n_{3}+n_{4}}}{\prod_{r \in \widehat{\mathcal{N}}_{3} \cup \mathcal{N}_{4}}\left(a_{u}-a_{q}\right)}$.

Having obtained the explicit first-order expression for $\delta \sigma^{(3)}$, we can use it in (100) and (101) to derive the firstorder expression for $\delta \sigma^{(2)}$. Explicitly we have

$\delta \sigma_{t}^{(2)}=\frac{(-1)^{n_{2}} \Lambda_{4 \mathrm{~d}}^{2 N}}{\Lambda_{1}^{n_{1}+n_{2}} \Lambda_{2}^{n_{2}+n_{3}} \Lambda_{3}^{n_{3}+n_{4}} \prod_{r \in \widehat{\mathcal{N}}_{1} \cup \mathcal{N}_{4}}\left(a_{t}-a_{r}\right)}$

for $t \in \mathcal{N}_{1}$, and

$\delta \sigma_{t}^{(2)}=\frac{(-1)^{n_{1}} \Lambda_{2}^{n_{2}+n_{3}}}{\prod_{r \in \widehat{\mathcal{N}}_{2} \cup \mathcal{N}_{3}}\left(a_{t}-a_{r}\right)}$ for $t \in \mathcal{N}_{2}$. Further substituting these results in (99), we get the first-order expression for $\delta \sigma^{(1)}$, namely

$$
\begin{aligned}
\delta \sigma_{s}^{(1)}= & \frac{\Lambda_{1}^{n_{1}+n_{2}}}{\prod_{r \in \widehat{\mathcal{N}}_{1} \cup \mathcal{N}_{2}}\left(a_{s}-a_{r}\right)} \\
& +\frac{(-1)^{n_{2}} \Lambda_{4 \mathrm{~d}}^{2 N}}{\Lambda_{1}^{n_{1}+n_{2}} \Lambda_{2}^{n_{2}+n_{3}} \Lambda_{3}^{n_{3}+n_{4}} \prod_{r \in \widehat{\mathcal{N}}_{1} \cup \mathcal{N}_{4}}\left(a_{s}-a_{r}\right)}
\end{aligned}
$$

for $s \in \mathcal{N}_{1}$.

Using this explicit solution in (29) and integrating in, we obtain that the twisted superpotential in the vacuum is given by

$$
\begin{aligned}
\left.\mathcal{W}\right|_{\sigma_{\star}}= & \sum_{s \in \mathcal{N}_{1}} \frac{\Lambda_{1}^{n_{1}+n_{2}}}{\prod_{r \in \widehat{\mathcal{N}}_{1} \cup \mathcal{N}_{2}}\left(a_{s}-a_{r}\right)} \\
& +\sum_{t \in \mathcal{N}_{2}} \frac{(-1)^{n_{1}} \Lambda_{2}^{n_{2}+n_{3}}}{\prod_{r \in \widehat{\mathcal{N}}_{2} \cup \mathcal{N}_{3}}\left(a_{t}-a_{r}\right)} \\
& +\sum_{u \in \mathcal{N}_{3}} \frac{(-1)^{n_{1}+n_{2}} \Lambda_{3}^{n_{3}+n_{4}}}{\prod_{r \in \widehat{\mathcal{N}}_{3} \cup \mathcal{N}_{4}}\left(a_{u}-a_{r}\right)} \\
& +\sum_{s \in \mathcal{N}_{1}} \frac{(-1)^{n_{2}+1} \Lambda_{4 \mathrm{~d}}^{2 N}}{\Lambda_{1}^{n_{1}+n_{2}} \Lambda_{2}^{n_{2}+n_{3}} \Lambda_{3}^{n_{3}+n_{4}} \prod_{r \in \mathcal{N}_{4} \cup \widehat{\mathcal{N}}_{1}}\left(a_{s}-a_{r}\right)}
\end{aligned}
$$

which, term by term, matches the localization result (90) if the $q$ vs $\Lambda$ map is

$$
\begin{aligned}
& q_{1}=(-1)^{n_{1}} \Lambda_{1}^{n_{1}+n_{2}}, \\
& q_{2}=(-1)^{n_{1}+n_{2}} \Lambda_{2}^{n_{2}+n_{3}}, \\
& q_{3}=(-1)^{n_{1}+n_{2}+n_{3}} \Lambda_{3}^{n_{3}+n_{4}}
\end{aligned}
$$

with $q_{1} q_{2} q_{3} q_{4}=(-1)^{N} \Lambda_{4 \mathrm{~d}}^{2 N}$.

\section{Quiver $Q_{1}$}

Let us now consider the quiver $Q_{1}$ :

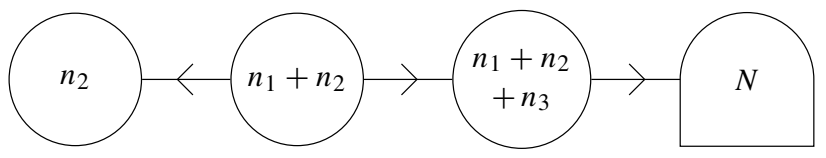

The corresponding twisted chiral ring equations are

$$
\begin{aligned}
\mathcal{Q}_{2}\left(\sigma_{s}^{(1)}\right)= & (-1)^{n_{1}+n_{2}} \Lambda_{1}^{n_{1}+n_{2}} \\
\mathcal{Q}_{1}\left(\sigma_{t}^{(2)}\right) \mathcal{Q}_{3}\left(\sigma_{t}^{(2)}\right)= & \Lambda_{2}^{n_{1}+2 n_{2}+n_{3}} \\
\mathcal{P}_{N}\left(\sigma_{u}^{(3)}\right)= & (-1)^{n_{1}+n_{2}}\left(\Lambda_{3}^{n_{3}+n_{4}} \mathcal{Q}_{2}\left(\sigma_{u}^{(3)}\right)\right. \\
& \left.+\frac{\Lambda_{4 \mathrm{~d}}^{2 N}}{\Lambda_{3}^{n_{3}+n_{4}} \mathcal{Q}_{2}\left(\sigma_{u}^{(3)}\right)}\right)
\end{aligned}
$$


for $s \in \mathcal{N}_{2}, t \in \mathcal{N}_{1} \cup \mathcal{N}_{2}$, and $u \in \mathcal{N}_{1} \cup \mathcal{N}_{2} \cup \mathcal{N}_{3}$, respectively. Here, to avoid clutter, we have denoted the low-energy scales $\Lambda_{I}^{Q_{1}}$ simply as $\Lambda_{I}$. The solution of these equations about the classical vacuum indicated in the second row of Table 1 is a generalization of what we have discussed in the previous subsection for the quiver $Q_{0}$, and thus we do not repeat it here. Instead, we write the result of substituting this solution into (29) and integrating in, which yields the twisted superpotential in the vacuum, namely

$$
\begin{aligned}
\left.\mathcal{W}\right|_{\sigma_{\star}}= & \sum_{t \in \mathcal{N}_{2}} \frac{(-1)^{n_{1}+n_{2}+1} \Lambda_{1}^{n_{1}+n_{2}}}{\prod_{r \in \mathcal{N}_{1} \cup \widehat{\mathcal{N}}_{2}}\left(a_{t}-a_{r}\right)} \\
& +\sum_{t \in \mathcal{N}_{2}} \frac{(-1)^{n_{1}+n_{2}+1} \Lambda_{2}^{n_{1}+2 n_{2}+n_{3}}}{\Lambda_{1}^{n_{1}+n_{2}} \prod_{r \in \widehat{\mathcal{N}}_{2} \cup \mathcal{N}_{3}}\left(a_{t}-a_{r}\right)} \\
& +\sum_{u \in \mathcal{N}_{3}} \frac{(-1)^{n_{1}+n_{2}} \Lambda_{3}^{n_{3}+n_{4}}}{\prod_{r \in \widehat{\mathcal{N}}_{3} \cup \mathcal{N}_{4}}\left(a_{u}-a_{r}\right)} \\
& +\sum_{s \in \mathcal{N}_{1}} \frac{(-1)^{n_{1}+n_{2}} \Lambda_{4 \mathrm{~d}}^{2 N}}{\Lambda_{2}^{n_{1}+2 n_{2}+n_{3}} \Lambda_{3}^{n_{3}+n_{4}} \prod_{r \in \mathcal{N}_{4} \cup \widehat{\mathcal{N}}_{1}}\left(a_{s}-a_{r}\right)} .
\end{aligned}
$$

It is easy to see that this exactly matches, term by term, the superpotential (91) obtained from localization, if the following $q$ vs $\Lambda$ map is used

$$
\begin{aligned}
& q_{1}=(-1)^{n_{2}} \Lambda_{1}^{n_{1}+n_{2}}, \\
& q_{2}=\frac{(-1)^{n_{1}+1} \Lambda_{2}^{n_{1}+2 n_{2}+n_{3}}}{\Lambda_{1}^{n_{1}+n_{2}}}, \\
& q_{3}=(-1)^{n_{1}+n_{2}+n_{3}} \Lambda_{3}^{n_{3}+n_{4}}
\end{aligned}
$$

with $q_{1} q_{2} q_{3} q_{4}=(-1)^{N} \Lambda_{4 \mathrm{~d}}^{2 N}$.

\section{Quiver $Q_{2}$}

We now consider the quiver $Q_{2}$, namely

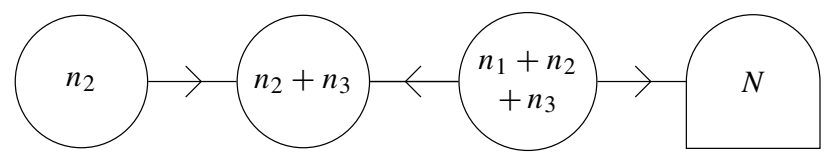

The corresponding twisted chiral ring equations are

$$
\begin{aligned}
\mathcal{Q}_{2}\left(\sigma_{s}^{(1)}\right) & =\Lambda_{1}^{n_{2}+n_{3}}, \\
\mathcal{Q}_{1}\left(\sigma_{t}^{(2)}\right) \mathcal{Q}_{3}\left(\sigma_{t}^{(2)}\right) & =(-1)^{n_{1}+n_{3}} \Lambda_{2}^{n_{1}+2 n_{2}+n_{3}}, \\
\mathcal{P}_{N}\left(\sigma_{u}^{(3)}\right) & =\frac{\Lambda_{3}^{N+n_{2}+n_{3}}}{\mathcal{Q}_{2}\left(\sigma_{u}^{(3)}\right)}+\frac{\Lambda_{4 \mathrm{~d}}^{2 N} \mathcal{Q}_{2}\left(\sigma_{u}^{(3)}\right)}{\Lambda_{3}^{N+n_{2}+n_{3}}} .
\end{aligned}
$$

for $s \in \mathcal{N}_{2}, t \in \mathcal{N}_{2} \cup \mathcal{N}_{3}$ and $u \in \mathcal{N}_{1} \cup \mathcal{N}_{2} \cup \mathcal{N}_{3}$, respectively. Again, to avoid clutter, we have denoted $\Lambda_{I}^{Q_{2}}$ simply as $\Lambda_{I}$.
Solving around the vacuum indicated in Table 1, plugging the solution into (29) and integrating in, we find

$$
\begin{aligned}
\left.\mathcal{W}\right|_{\sigma_{\star}}= & \sum_{t \in \mathcal{N}_{2}} \frac{(-1)^{n_{1}+n_{3}} \Lambda_{2}^{n_{1}+2 n_{2}+n_{3}}}{\Lambda_{1}^{n_{2}+n_{3}} \prod_{r \in \mathcal{N}_{1} \cup \widehat{\mathcal{N}}_{2}}\left(a_{t}-a_{r}\right)} \\
& +\sum_{t \in \mathcal{N}_{2}} \frac{\Lambda_{1}^{n_{2}+n_{3}}}{\prod_{r \in \widehat{\mathcal{N}}_{2} \cup \mathcal{N}_{3}}\left(a_{t}-a_{r}\right)} \\
& +\sum_{u \in \mathcal{N}_{3}} \frac{(-1)^{n_{1}+n_{3}+1} \Lambda_{3}^{N+n_{2}+n_{3}}}{\Lambda_{2}^{n_{1}+2 n_{2}+n_{3}} \prod_{r \in \widehat{\mathcal{N}}_{3} \cup \mathcal{N}_{4}}\left(a_{u}-a_{r}\right)} \\
& -\sum_{s \in \mathcal{N}_{1}} \frac{\Lambda_{4 \mathrm{~d}}^{2 N}}{\Lambda_{3}^{N+n_{2}+n_{3}} \prod_{r \in \mathcal{N}_{4} \cup \widehat{\mathcal{N}}_{1}}\left(a_{s}-a_{r}\right)} .
\end{aligned}
$$

This agrees, term by term, with the localization result (91) if the following $q$ vs $\Lambda$ map is used

$$
\begin{aligned}
& q_{1}=(-1)^{n_{3}+1} \frac{\Lambda_{2}^{n_{1}+2 n_{2}+n_{3}}}{\Lambda_{1}^{n_{2}+n_{3}}}, \\
& q_{2}=(-1)^{n_{2}} \Lambda_{1}^{n_{2}+n_{3}}, \\
& q_{3}=(-1)^{n_{1}+1} \frac{\Lambda_{3}^{N+n_{2}+n_{3}}}{\Lambda_{2}^{n_{1}+2 n_{2}+n_{3}}}
\end{aligned}
$$

with $q_{1} q_{2} q_{3} q_{4}=(-1)^{N} \Lambda_{4 \mathrm{~d}}^{2 N}$.

Quiver $Q_{4}$

The quiver $Q_{4}$ is

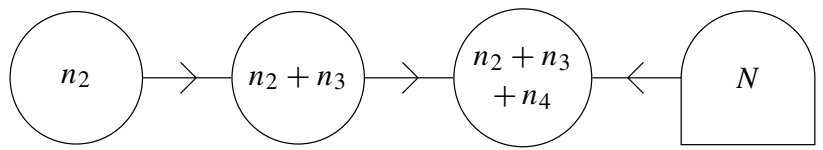

and the corresponding twisted chiral ring equations are

$$
\begin{aligned}
\mathcal{Q}_{2}\left(\sigma_{s}^{(1)}\right)= & \Lambda_{1}^{n_{2}+n_{3}}, \\
\mathcal{Q}_{3}\left(\sigma_{t}^{(2)}\right)= & (-1)^{n_{2}} \Lambda_{2}^{n_{3}+n_{4}} \mathcal{Q}_{1}\left(\sigma_{t}^{(2)}\right), \\
\mathcal{P}_{N}\left(\sigma_{u}^{(3)}\right)= & (-1)^{n_{1}+n_{4}}\left(\frac{\Lambda_{3}^{N+n_{2}+n_{3}}}{\mathcal{Q}_{2}\left(\sigma_{u}^{(3)}\right)}\right. \\
& \left.+\frac{\Lambda_{4 \mathrm{~d}}^{2 N} \mathcal{Q}_{2}\left(\sigma_{u}^{(3)}\right)}{\Lambda_{3}^{N+n_{2}+n_{3}}}\right)
\end{aligned}
$$

for $s \in \mathcal{N}_{2}, t \in \mathcal{N}_{2} \cup \mathcal{N}_{3}$ and $u \in \mathcal{N}_{2} \cup \mathcal{N}_{3} \cup \mathcal{N}_{4}$. Again we have denoted the low-energy scales $\Lambda_{I}^{Q_{4}}$ simply as $\Lambda_{I}$. Proceeding as discussed above, in this case we find

$$
\begin{aligned}
\left.\mathcal{W}\right|_{\sigma_{\star}}= & \sum_{t \in \mathcal{N}_{2}} \frac{(-1)^{n_{1}+n_{2}+n_{4}+1} \Lambda_{3}^{N+n_{2}+n_{3}}}{\Lambda_{1}^{n_{2}+n_{3}} \Lambda_{2}^{n_{3}+n_{4}} \prod_{r \in \mathcal{N}_{1} \cup \widehat{\mathcal{N}}_{2}}\left(a_{t}-a_{r}\right)} \\
& +\sum_{t \in \mathcal{N}_{2}} \frac{\Lambda_{1}^{n_{2}+n_{3}}}{\prod_{r \in \widehat{\mathcal{N}}_{2} \cup \mathcal{N}_{3}}\left(a_{t}-a_{r}\right)}
\end{aligned}
$$




$$
\begin{aligned}
& +\sum_{u \in \mathcal{N}_{3}} \frac{(-1)^{n_{2}} \Lambda_{2}^{n_{3}+n_{4}}}{\prod_{r \in \widehat{\mathcal{N}}_{3} \cup \mathcal{N}_{4}}\left(a_{u}-a_{r}\right)} \\
& +\sum_{v \in \mathcal{N}_{4}} \frac{(-1)^{n_{1}+n_{4}} \Lambda_{4 \mathrm{~d}}^{2 N}}{\Lambda_{3}^{N+n_{2}+n_{3}} \prod_{r \in \widehat{\mathcal{N}}_{4} \cup \mathcal{N}_{1}}\left(a_{v}-a_{r}\right)} .
\end{aligned}
$$

This expression exactly matches, term by term, with the localization result (92) provided the following $q$ vs $\Lambda$ map is used:

$$
\begin{aligned}
& q_{1}=(-1)^{n_{2}+n_{4}} \frac{\Lambda_{3}^{N+n_{2}+n_{3}}}{\Lambda_{1}^{n_{2}+n_{3}} \Lambda_{2}^{n_{3}+n_{4}}}, \\
& q_{2}=(-1)^{n_{2}} \Lambda_{1}^{n_{2}+n_{3}}, \\
& q_{3}=(-1)^{n_{2}+n_{3}} \Lambda_{2}^{n_{3}+n_{4}}
\end{aligned}
$$

with $q_{1} q_{2} q_{3} q_{4}=(-1)^{N} \Lambda_{4 \mathrm{~d}}^{2 N}$.

\section{Quiver $Q_{5}$}

The quiver $Q_{5}$ is

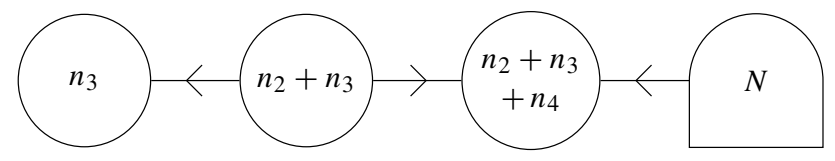

and the corresponding twisted chiral ring equations are

$$
\begin{aligned}
\mathcal{Q}_{2}\left(\sigma_{s}^{(1)}\right)= & (-1)^{n_{2}+n_{3}} \Lambda_{1}^{n_{2}+n_{3}}, \\
\mathcal{Q}_{1}\left(\sigma_{t}^{(2)}\right) \mathcal{Q}_{3}\left(\sigma_{t}^{(2)}\right)= & \Lambda_{2}^{n_{2}+2 n_{3}+n_{4}}, \\
\mathcal{P}_{N}\left(\sigma_{u}^{(3)}\right)= & (-1)^{n_{1}+n_{4}}\left(\frac{\Lambda_{3}^{N+n_{2}+n_{3}}}{\mathcal{Q}_{2}\left(\sigma_{u}^{(3)}\right)}\right. \\
& \left.+\frac{\Lambda_{4 \mathrm{~d}}^{2 N} \mathcal{Q}_{2}\left(\sigma_{u}^{(3)}\right)}{\Lambda_{3}^{N+n_{2}+n_{3}}}\right)
\end{aligned}
$$

for $s \in \mathcal{N}_{3}, t \in \mathcal{N}_{2} \cup \mathcal{N}_{3}$ and $u \in \mathcal{N}_{2} \cup \mathcal{N}_{3} \cup \mathcal{N}_{4}$ respectively. As before, to avoid clutter we have denoted $\Lambda_{I}^{Q_{5}}$ simply as $\Lambda_{I}$. Solving these equations around the appropriate vacuum (see Table 1), using (29) and integrating in, we find

$$
\begin{aligned}
\left.\mathcal{W}\right|_{\sigma_{\star}}= & \sum_{t \in \mathcal{N}_{2}} \frac{(-1)^{n_{1}+n_{4}} \Lambda_{3}^{N+n_{2}+n_{3}}}{\Lambda_{2}^{n_{2}+2 n_{3}+n_{4}} \prod_{r \in \mathcal{N}_{1} \cup \widehat{\mathcal{N}}_{2}}\left(a_{t}-a_{r}\right)} \\
& +\sum_{u \in \mathcal{N}_{3}} \frac{(-1)^{n_{2}+n_{3}+1} \Lambda_{1}^{n_{2}+n_{3}}}{\prod_{r \in \mathcal{N}_{2} \cup \widehat{\mathcal{N}}_{3}}\left(a_{u}-a_{r}\right)} \\
& +\sum_{u \in \mathcal{N}_{3}} \frac{(-1)^{n_{2}+n_{3}+1} \Lambda_{3}^{N+n_{2}+n_{3}}}{\Lambda_{1}^{n_{1}+n_{3}} \prod_{r \in \widehat{\mathcal{N}_{3}} \cup \mathcal{N}_{4}}\left(a_{u}-a_{r}\right)} \\
& +\sum_{v \in \mathcal{N}_{4}} \frac{(-1)^{n_{1}+n_{4}} \Lambda_{4 \mathrm{~d}}^{2 N}}{\Lambda_{3}^{N+n_{2}+n_{3}} \prod_{r \in \widehat{\mathcal{N}}_{4} \cup \mathcal{N}_{1}}\left(a_{v}-a_{r}\right)} .
\end{aligned}
$$

This expression agrees, term by term, with the localization result (93) if the following $q$ vs $\Lambda$ map is used:

$$
\begin{aligned}
& q_{1}=(-1)^{n_{4}+1} \frac{\Lambda_{3}^{N+n_{2}+n_{3}}}{\Lambda_{2}^{n_{2}+2 n_{3}+n_{4}}}, \\
& q_{2}=(-1)^{n_{3}} \Lambda_{1}^{n_{2}+n_{3}}, \\
& q_{3}=(-1)^{n_{2}+1} \frac{\Lambda_{2}^{n_{2}+2 n_{3}+n_{4}}}{\Lambda_{1}^{n_{2}+n_{3}}}
\end{aligned}
$$

with $q_{1} q_{2} q_{3} q_{4}=(-1)^{N} \Lambda_{4 \mathrm{~d}}^{2 N}$.

\section{Quiver $Q_{6}$}

The quiver $Q_{6}$ is

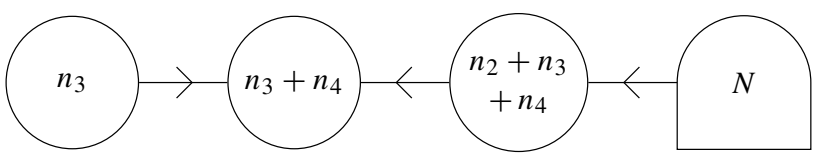

and the corresponding twisted chiral ring equations are

$$
\begin{aligned}
\mathcal{Q}_{2}\left(\sigma_{s}^{(1)}\right) & =\Lambda_{1}^{n_{3}+n_{4}} \\
\mathcal{Q}_{1}\left(\sigma_{t}^{(2)}\right) \mathcal{Q}_{3}\left(\sigma_{t}^{(2)}\right)= & (-1)^{n_{2}+n_{4}} \Lambda_{2}^{n_{2}+2 n_{3}+n_{4}} \\
\mathcal{P}_{N}\left(\sigma_{u}^{(3)}\right)= & (-1)^{N}\left(\Lambda_{3}^{n_{1}+n_{2}} \mathcal{Q}_{2}\left(\sigma_{u}^{(3)}\right)\right. \\
& \left.+\frac{\Lambda_{4 \mathrm{~d}}^{2 N}}{\Lambda_{3}^{n_{1}+n_{2}} \mathcal{Q}_{2}\left(\sigma_{u}^{(3)}\right)}\right)
\end{aligned}
$$

with $s \in \mathcal{N}_{3}, t \in \mathcal{N}_{3} \cup \mathcal{N}_{4}$ and $u \in \mathcal{N}_{2} \cup \mathcal{N}_{3} \cup \mathcal{N}_{4}$ respectively. Again the low-energy scales of this quiver have been denoted simply as $\Lambda_{I}$ instead of $\Lambda_{I}^{Q_{6}}$. We solve these equations about the classical vacuum given in Table 1; after inserting the solution in (29) and integrating in, we obtain

$$
\begin{aligned}
\left.\mathcal{W}\right|_{\sigma_{\star}}= & \sum_{t \in \mathcal{N}_{2}} \frac{(-1)^{N+1} \Lambda_{3}^{n_{1}+n_{2}}}{\prod_{r \in \mathcal{N}_{1} \cup \widehat{\mathcal{N}}_{2}}\left(a_{t}-a_{r}\right)} \\
& +\sum_{u \in \mathcal{N}_{3}} \frac{(-1)^{n_{2}+n_{4}} \Lambda_{2}^{n_{2}+2 n_{3}+n_{4}}}{\Lambda_{1}^{n_{3}+n_{4}} \prod_{r \in \mathcal{N}_{2} \cup \widehat{\mathcal{N}}_{3}}\left(a_{u}-a_{r}\right)} \\
& +\sum_{u \in \mathcal{N}_{3}} \frac{\Lambda_{1}^{n_{3}+n_{4}}}{\prod_{r \in \widehat{\mathcal{N}}_{3} \cup \mathcal{N}_{4}}\left(a_{u}-a_{r}\right)} \\
& +\sum_{v \in \mathcal{N}_{4}} \frac{(-1)^{n_{1}+n_{3}+1} \Lambda_{4 \mathrm{~d}}^{2 N}}{\Lambda_{2}^{n_{2}+2 n_{3}+n_{4}} \Lambda_{3}^{n_{1}+n_{2}} \prod_{r \in \widehat{\mathcal{N}}_{4} \cup \mathcal{N}_{1}}\left(a_{v}-a_{r}\right)}
\end{aligned}
$$


This expression perfectly matches, term by term, the localization result (93) if the $q$ vs $\Lambda$ map is

$q_{1}=(-1)^{n_{2}+n_{3}+n_{4}} \Lambda_{3}^{n_{1}+n_{2}}$,

$q_{2}=(-1)^{n_{4}+1} \frac{\Lambda_{2}^{n_{2}+2 n_{3}+n_{4}}}{\Lambda_{1}^{n_{3}+n_{4}}}$,

$q_{3}=(-1)^{n_{3}} \Lambda_{1}^{n_{3}+n_{4}}$

with $q_{1} q_{2} q_{3} q_{4}=(-1)^{N} \Lambda_{4 \mathrm{~d}}^{2 N}$.

Quiver $Q_{7}$

The last quiver of the duality chain of in Fig. 8 is

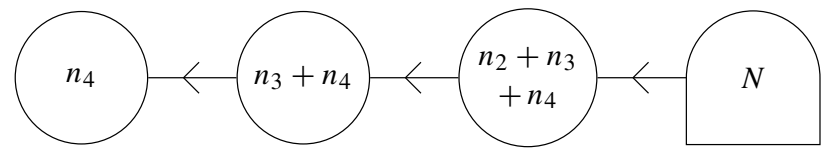

and the associated twisted chiral ring equations are

$$
\begin{aligned}
\mathcal{Q}_{2}\left(\sigma_{s}^{(1)}\right)= & (-1)^{n_{3}+n_{4}} \Lambda_{1}^{n_{3}+n_{4}}, \\
\mathcal{Q}_{3}\left(\sigma_{t}^{(2)}\right)= & (-1)^{n_{2}+n_{3}+n_{4}} \Lambda_{2}^{n_{2}+n_{3}} \mathcal{Q}_{1}\left(\sigma_{t}^{(2)}\right), \\
\mathcal{P}_{N}\left(\sigma_{u}^{(3)}\right)= & (-1)^{N}\left(\Lambda_{3}^{n_{1}+n_{2}} \mathcal{Q}_{2}\left(\sigma_{u}^{(3)}\right)\right. \\
& \left.+\frac{\Lambda_{4 \mathrm{~d}}^{2 N}}{\Lambda_{3}^{n_{1}+n_{2}} \mathcal{Q}_{2}\left(\sigma_{u}^{(3)}\right)}\right)
\end{aligned}
$$

with $s \in \mathcal{N}_{4}, t \in \mathcal{N}_{3} \cup \mathcal{N}_{4}$ and $u \in \mathcal{N}_{2} \cup \mathcal{N}_{3} \cup \mathcal{N}_{4}$. Here, $\Lambda_{I}$ denote the scales of this quiver. Solving these equation around the vacuum reported in the last row of Table 1, and proceeding as in the previous cases, we obtain

$$
\begin{aligned}
\left.\mathcal{W}\right|_{\sigma_{\star}}= & \sum_{t \in \mathcal{N}_{2}} \frac{(-1)^{N+1} \Lambda_{3}^{n_{1}+n_{2}}}{\prod_{r \in \mathcal{N}_{1} \cup \widehat{\mathcal{N}}_{2}}\left(a_{t}-a_{r}\right)} \\
& +\sum_{u \in \mathcal{N}_{3}} \frac{(-1)^{n_{2}+n_{3}+n_{4}+1} \Lambda_{2}^{n_{2}+n_{3}}}{\prod_{r \in \mathcal{N}_{2} \cup \widehat{\mathcal{N}}_{3}}\left(a_{u}-a_{r}\right)} \\
& +\sum_{v \in \mathcal{N}_{4}} \frac{(-1)^{n_{3}+n_{4}+1} \Lambda_{1}^{n_{3}+n_{4}}}{\prod_{r \in \mathcal{N}_{3} \cup \widehat{\mathcal{N}}_{4}}\left(a_{u}-a_{r}\right)} \\
& +\sum_{v \in \mathcal{N}_{4}} \frac{(-1)^{n_{1}+n_{3}+n_{4}} \Lambda_{4 \mathrm{~d}}^{2 N}}{\Lambda_{1}^{n_{3}+n_{4}} \Lambda_{2}^{n_{2}+n_{3}} \Lambda_{3}^{n_{1}+n_{2}} \prod_{r \in \widehat{\mathcal{N}}_{4} \cup \mathcal{N}_{1}}\left(a_{v}-a_{r}\right)} .
\end{aligned}
$$

This agrees, term by term, with the localization result (94) using the following $q$ vs $\Lambda$ map

$$
\begin{aligned}
& q_{1}=(-1)^{n_{2}+n_{3}+n_{4}}\left(\Lambda_{3}\right)^{n_{1}+n_{2}}, \\
& q_{2}=(-1)^{n_{3}+n_{4}}\left(\Lambda_{2}\right)^{n_{2}+n_{3}}, \\
& q_{3}=(-1)^{n_{4}}\left(\Lambda_{1}\right)^{n_{3}+n_{4}}
\end{aligned}
$$

with $q_{1} q_{2} q_{3} q_{4}=(-1)^{N} \Lambda_{4 \mathrm{~d}}^{2 N}$.

Quiver $\widehat{Q}_{1}$

The non-linear quiver $\widehat{Q}_{1}$ appearing in the duality chain represented in Fig. 9 is

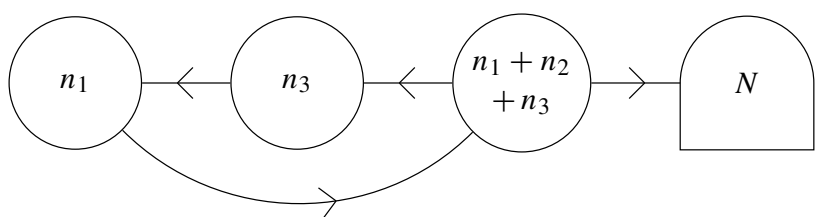

and its corresponding twisted chiral ring equations are

$$
\begin{aligned}
\mathcal{Q}_{3}\left(\sigma_{s}^{(1)}\right)= & (-1)^{n_{3}} \Lambda_{1}^{n_{1}+n_{2}} \mathcal{Q}_{2}\left(\sigma_{s}^{(1)}\right) \\
\mathcal{Q}_{3}\left(\sigma_{t}^{(2)}\right)= & (-1)^{n_{1}+n_{2}+n_{3}} \Lambda_{2}^{n_{2}+n_{3}} \mathcal{Q}_{1}\left(\sigma_{t}^{(2)}\right) \\
\mathcal{P}_{N}\left(\sigma_{u}^{(3)}\right)= & (-1)^{n_{1}}\left(\frac{\Lambda_{3}^{N+n_{3}-n_{1}} \mathcal{Q}_{1}\left(\sigma_{u}^{(3)}\right)}{\mathcal{Q}_{2}\left(\sigma_{u}^{(3)}\right)}\right. \\
& \left.+\frac{\Lambda_{4 \mathrm{~d}}^{2 N} \mathcal{Q}_{2}\left(\sigma_{u}^{(3)}\right)}{\Lambda_{3}^{N+n_{3}-n_{1}} \mathcal{Q}_{1}\left(\sigma_{u}^{(3)}\right)}\right)
\end{aligned}
$$

for $s \in \mathcal{N}_{1}, t \in \mathcal{N}_{3}$ and $u \in \mathcal{N}_{1} \cup \mathcal{N}_{2} \cup \mathcal{N}_{3}$. Again we have denoted the low-energy scales $\Lambda_{I}^{\widehat{Q}_{1}}$ simply as $\Lambda_{I}$. Solving these equations around the vacuum given in the first row of Table 5, using (29) and integrating in, we obtain

$$
\begin{aligned}
\left.\mathcal{W}\right|_{\sigma_{\star}}=\sum_{s \in \mathcal{N}_{1}} \frac{(-1)^{n_{3}} \Lambda_{1}^{n_{1}+n_{2}}}{\prod_{r \in \widehat{\mathcal{N}}_{1} \cup \mathcal{N}_{2}}\left(a_{s}-a_{r}\right)} \\
+\sum_{u \in \mathcal{N}_{3}} \frac{(-1)^{n_{1}+n_{2}+n_{3}+1} \Lambda_{2}^{n_{2}+n_{3}}}{\prod_{r \in \mathcal{N}_{2} \cup \widehat{\mathcal{N}}_{3}}\left(a_{u}-a_{r}\right)} \\
\quad+\sum_{u \in \mathcal{N}_{3}} \frac{(-1)^{n_{2}+n_{3}+1} \Lambda_{3}^{n_{2}+2 n_{3}+n_{4}}}{\Lambda_{2}^{n_{2}+n_{3}} \prod_{r \in \widehat{\mathcal{N}}_{3} \cup \mathcal{N}_{4}}\left(a_{u}-a_{r}\right)} \\
+\sum_{s \in \mathcal{N}_{1}} \frac{(-1)^{n_{1}+n_{3}} \Lambda_{4 \mathrm{~d}}^{2 N}}{\Lambda_{1}^{n_{1}+n_{2}} \Lambda_{3}^{n_{2}+2 n_{3}+n_{4}} \prod_{r \in \mathcal{N}_{4} \cup \widehat{\mathcal{N}}_{1}}\left(a_{s}-a_{r}\right)} .
\end{aligned}
$$

This matches, term by term, the localization result (95) using the following $q$ vs $\Lambda$ map:

$$
\begin{aligned}
& q_{1}=(-1)^{n_{1}+n_{3}} \Lambda_{1}^{n_{1}+n_{2}}, \\
& q_{2}=(-1)^{n_{1}+n_{3}} \Lambda_{2}^{n_{2}+n_{3}}, \\
& q_{3}=(-1)^{n_{2}+1} \frac{\Lambda_{3}^{n_{2}+2 n_{3}+n_{4}}}{\Lambda_{2}^{n_{2}+n_{3}}}
\end{aligned}
$$

with $q_{1} q_{2} q_{3} q_{4}=(-1)^{N} \Lambda_{4 \mathrm{~d}}^{2 N}$. 
Quiver $Q_{3}$

The quiver $Q_{3}$ is

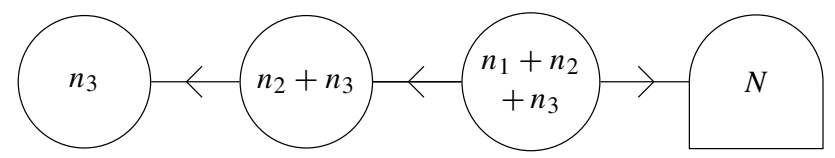

and its twisted chiral ring equations are

$$
\begin{aligned}
& \mathcal{Q}_{2}\left(\sigma_{s}^{(1)}\right)=(-1)^{n_{2}+n_{3}} \Lambda_{1}^{n_{2}+n_{3}}, \\
& \mathcal{Q}_{3}\left(\sigma_{t}^{(2)}\right)=(-1)^{n_{1}+n_{2}+n_{3}} \Lambda_{2}^{n_{1}+n_{2}} \mathcal{Q}_{1}\left(\sigma_{t}^{(2)}\right), \\
& \mathcal{P}_{N}\left(\sigma_{u}^{(3)}\right)=\frac{\Lambda_{3}^{N+n_{2}+n_{3}}}{\mathcal{Q}_{2}\left(\sigma_{u}^{(3)}\right)}+\frac{\Lambda_{4 \mathrm{~d}}^{2 N} \mathcal{Q}_{2}\left(\sigma_{u}^{(3)}\right)}{\Lambda_{3}^{N+n_{2}+n_{3}}}
\end{aligned}
$$

for $s \in \mathcal{N}_{3}, t \in \mathcal{N}_{2} \cup \mathcal{N}_{3}$ and $u \in \mathcal{N}_{1} \cup \mathcal{N}_{2} \cup \mathcal{N}_{3}$, respectively. Again, $\Lambda_{I}$ denote the low-energy scales of this quiver. Solving these equations around the vacuum displayed in the middle row of Table 5 and proceeding in the usual way, we get

$$
\begin{aligned}
\left.\mathcal{W}\right|_{\sigma_{\star}}= & \sum_{t \in \mathcal{N}_{2}} \frac{(-1)^{n_{1}+n_{2}+n_{3}+1} \Lambda_{2}^{n_{1}+n_{2}}}{\prod_{r \in \mathcal{N}_{1} \cup \widehat{\mathcal{N}}_{2}}\left(a_{t}-a_{r}\right)} \\
& +\sum_{u \in \mathcal{N}_{3}} \frac{(-1)^{n_{2}+n_{3}+1} \Lambda_{1}^{n_{2}+n_{3}}}{\prod_{r \in \mathcal{N}_{2} \cup \widehat{\mathcal{N}}_{3}}\left(a_{u}-a_{r}\right)} \\
& +\sum_{u \in \mathcal{N}_{3}} \frac{(-1)^{n_{1}} \Lambda_{3}^{n_{2}+n_{3}+N}}{\Lambda_{1}^{n_{2}+n_{3}} \Lambda_{2}^{n_{1}+n_{2}} \prod_{r \in \widehat{\mathcal{N}}_{3} \cup \mathcal{N}_{4}}\left(a_{u}-a_{r}\right)} \\
& -\sum_{s \in \mathcal{N}_{1}} \frac{\Lambda_{4 \mathrm{~d}}^{2 N}}{\Lambda_{3}^{n_{2}+n_{3}+N} \prod_{r \in \mathcal{N}_{4} \cup \widehat{\mathcal{N}}_{1}}\left(a_{s}-a_{r}\right)}
\end{aligned}
$$

This matches, term by term, the localization result (96) using the following $q$ vs $\Lambda$ map:

$$
\begin{aligned}
& q_{1}=(-1)^{n_{2}+n_{3}} \Lambda_{2}^{n_{1}+n_{2}}, \\
& q_{2}=(-1)^{n_{3}} \Lambda_{1}^{n_{2}+n_{3}}, \\
& q_{3}=(-1)^{n_{1}+n_{3}} \frac{\Lambda_{3}^{n_{2}+n_{3}+N}}{\Lambda_{1}^{n_{2}+n_{3}} \Lambda_{2}^{n_{1}+n_{2}}}
\end{aligned}
$$

with $q_{1} q_{2} q_{3} q_{4}=(-1)^{N} \Lambda_{4 \mathrm{~d}}^{2 N}$.

Quiver $\widehat{Q}_{5}$

The second non-linear quiver in the duality chain of Fig. 9 is

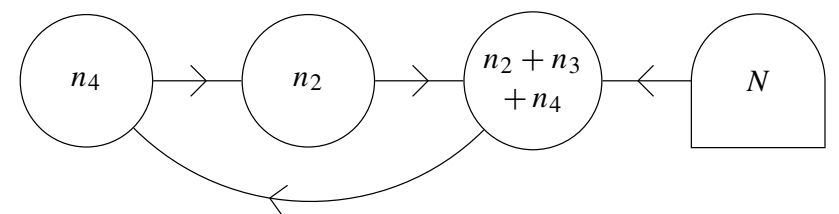

and the corresponding twisted chiral ring equations are

$$
\begin{aligned}
\mathcal{Q}_{3}\left(\sigma_{s}^{(1)}\right)= & (-1)^{n_{2}+n_{3}+n_{4}} \Lambda_{1}^{n_{3}+n_{4}} \mathcal{Q}_{2}\left(\sigma_{s}^{(1)}\right), \\
\mathcal{Q}_{3}\left(\sigma_{t}^{(2)}\right)= & (-1)^{n_{4}} \Lambda_{2}^{n_{2}+n_{3}} \mathcal{Q}_{1}\left(\sigma_{t}^{(2)}\right), \\
\mathcal{P}_{N}\left(\sigma_{u}^{(3)}\right)= & (-1)^{n_{1}+n_{3}+n_{4}}\left(\frac{\Lambda_{3}^{n_{1}+2 n_{2}+n_{3}} \mathcal{Q}_{1}\left(\sigma_{u}^{(3)}\right)}{\mathcal{Q}_{2}\left(\sigma_{u}^{(3)}\right)}\right. \\
& \left.+\frac{\Lambda_{4 \mathrm{~d}}^{2 N} \mathcal{Q}_{2}\left(\sigma_{u}^{(3)}\right)}{\Lambda_{3}^{n_{1}+2 n_{2}+n_{3}} \mathcal{Q}_{1}\left(\sigma_{u}^{(3)}\right)}\right)
\end{aligned}
$$

for $s \in \mathcal{N}_{4}, t \in \mathcal{N}_{2}$ and $u \in \mathcal{N}_{2} \cup \mathcal{N}_{3} \cup \mathcal{N}_{4}$, respectively. As usual, we have denoted the scales $\Lambda_{I}^{\widehat{Q}_{5}}$ simply as $\Lambda_{I}$. Solving these equations around the vacuum indicated in the last row of Table 5, using (29) and integrating in, we find

$$
\begin{aligned}
\left.\mathcal{W}\right|_{\sigma_{\star}}=\sum_{t \in \mathcal{N}_{2}} \frac{(-1)^{n_{1}+n_{3}} \Lambda_{3}^{n_{1}+2 n_{2}+n_{3}}}{\Lambda_{2}^{n_{2}+n_{3}} \prod_{r \in \mathcal{N}_{1} \cup \widehat{\mathcal{N}}_{2}}\left(a_{t}-a_{r}\right)} \\
\quad+\sum_{t \in \mathcal{N}_{2}} \frac{(-1)^{n_{4}} \Lambda_{2}^{n_{2}+n_{3}}}{\prod_{r \in \widehat{\mathcal{N}}_{2} \cup \mathcal{N}_{3}}\left(a_{t}-a_{r}\right)} \\
\quad+\sum_{v \in \mathcal{N}_{4}} \frac{(-1)^{n_{2}+n_{3}+n_{4}+1} \Lambda_{1}^{n_{3}+n_{4}}}{\prod_{r \in \mathcal{N}_{3} \cup \widehat{\mathcal{N}}_{4}}\left(a_{v}-a_{r}\right)} \\
\quad+\sum_{v \in \mathcal{N}_{4}} \frac{(-1)^{n_{1}+n_{2}+1} \Lambda_{4 \mathrm{~d}}^{2 N}}{\Lambda_{1}^{n_{3}+n_{4}} \Lambda_{3}^{n_{1}+2 n_{2}+n_{3}} \prod_{r \in \widehat{\mathcal{N}}_{4} \cup \mathcal{N}_{1}}\left(a_{v}-a_{r}\right)}
\end{aligned}
$$

This superpotential agrees, term by term, with the localization result (97) if the $q$ vs $\Lambda$ map is

$$
\begin{aligned}
& q_{1}=(-1)^{n_{3}+1} \frac{\Lambda_{3}^{n_{1}+2 n_{2}+n_{3}}}{\Lambda_{2}^{n_{2}+n_{3}}}, \\
& q_{2}=(-1)^{n_{2}+n_{4}} \Lambda_{2}^{n_{2}+n_{3}}, \\
& q_{3}=(-1)^{n_{2}+n_{4}} \Lambda_{1}^{n_{3}+n_{4}} \\
& \text { with } q_{1} q_{2} q_{3} q_{4}=(-1)^{N} \Lambda_{4 \mathrm{~d}}^{2 N} .
\end{aligned}
$$

\section{Some two-instanton results}

In this appendix we illustrate how the JK prescription works at two-instantons. In order to keep things as simple as possible, we just focus on the term in the superpotential that is proportional to $q_{1} q_{2}$. After using (8) and (9), it is not difficult to realize that this term takes the following form

$$
\begin{aligned}
& q_{1} q_{2} \lim _{\epsilon_{1}, \hat{\epsilon}_{2} \rightarrow 0} \int \frac{d \chi_{1}}{2 \pi \mathrm{i}} \frac{d \chi_{2}}{2 \pi \mathrm{i}} \frac{1}{\left(\chi_{1}-\chi_{2}+\hat{\epsilon}_{2}\right)} \\
& \times \prod_{s \in \mathcal{N}_{1}} \frac{1}{\left(a_{s}-\chi_{1}+\frac{1}{2}\left(\epsilon_{1}+\hat{\epsilon}_{2}\right)\right)}
\end{aligned}
$$


Table 8 We list the poles that contribute to the $q_{1} q_{2}$ term of the superpotential for the quivers $Q_{1}$ and $Q_{2}$. In the second column we have shown only the parts of the JK vector that are relevant for this two-instanton contribution

\begin{tabular}{llll}
\hline Quiver & JK vector & Poles & \\
\hline$Q_{1}$ & $-\zeta_{1}^{Q_{1}} \chi_{1}-\zeta_{2}^{Q_{1}} \chi_{2}$ & $\chi_{1}=a_{t}-\frac{1}{2}\left(\epsilon_{1}+\hat{\epsilon}_{2}\right)$ & $t \in \mathcal{N}_{2}$ \\
& & $\chi_{2}=a_{t}+\frac{1}{2}\left(\epsilon_{1}+\hat{\epsilon}_{2}\right)$ & $t \in \mathcal{N}_{2}$ \\
& $\chi_{1}=a_{s}+\frac{1}{2}\left(\epsilon_{1}+\hat{\epsilon}_{2}\right)$ & $s \in \mathcal{N}_{1}$ \\
& $\chi_{2}=\chi_{1}+\hat{\epsilon}_{2}$ & \\
& $\chi_{1}=\chi_{2}-\hat{\epsilon}_{2}$ & \\
& $\chi_{2}=a_{t}+\frac{1}{2}\left(\epsilon_{1}+\hat{\epsilon}_{2}\right)$ & $t \in \mathcal{N}_{2}$ \\
& $\chi_{2}=a_{t}+\frac{1}{2}\left(\epsilon_{1}+\hat{\epsilon}_{2}\right)$ & $t \in \mathcal{N}_{2}$ \\
& & $\chi_{1}=a_{t}-\frac{1}{2}\left(\epsilon_{1}+\hat{\epsilon}_{2}\right)$ & $t \in \mathcal{N}_{2}$ \\
& $\chi_{1}^{Q_{2}} \chi_{2}-\zeta_{2}^{Q_{2}} \chi_{1}$ & $\chi_{1}+\hat{\epsilon}_{2}$ & \\
& & $\chi_{1}=a_{t}-\frac{1}{2}\left(\epsilon_{1}+\hat{\epsilon}_{2}\right)$ & $t \in \mathcal{N}_{2}$ \\
& $\chi_{2}=a_{u}-\frac{1}{2}\left(\epsilon_{1}+\hat{\epsilon}_{2}\right)$ & $u \in \mathcal{N}_{3}$ \\
& $\chi_{1}=\chi_{2}-\hat{\epsilon}_{2}$ & \\
\hline & &
\end{tabular}

$$
\begin{aligned}
& \times \prod_{t \in \mathcal{N}_{2}} \frac{1}{\left(-a_{t}+\chi_{1}+\frac{1}{2}\left(\epsilon_{1}+\hat{\epsilon}_{2}\right)\right)\left(a_{t}-\chi_{2}+\frac{1}{2}\left(\epsilon_{1}+\hat{\epsilon}_{2}\right)\right)} \\
& \times \prod_{u \in \mathcal{N}_{3}} \frac{1}{\left(-a_{u}+\chi_{2}+\frac{1}{2}\left(\epsilon_{1}+\hat{\epsilon}_{2}\right)\right)} .
\end{aligned}
$$

We now apply the JK prescription to compute the double integral over $\chi_{1}$ and $\chi_{2}$. This amounts to choose two linear factors from the denominator, one containing $\chi_{1}$ and one containing $\chi_{2}$, such that the reference $\mathrm{JK}$ vector belongs to the cone defined by the chosen factors. Notice that this way of selecting the residues does not use any information on the $\Omega$-deformation parameters. In Table 8 we list the poles that are selected by this $\mathrm{JK}$ prescription for the quivers $Q_{1}$ and $Q_{2}$ of the duality chain of Fig. 8 .

An important point that we emphasized in the main body of the paper is that the $\mathrm{JK}$ vectors $-\zeta_{1}^{Q_{1}} \chi_{1}-\zeta_{2}^{Q_{1}} \chi_{2}$ and $-\zeta_{1}^{Q_{2}} \chi_{2}-\zeta_{2}^{Q_{2}} \chi_{1}$ pick up different sets of poles from the localization integrand, as a consequence of the different signs and magnitudes of the FI parameters. One can see this explicitly from the entries in the third column of Table 8 .

By calculating the residues over the poles selected by the JK vector $\eta_{Q_{1}}$ associated to the quiver $Q_{1}$, we find that the corresponding contribution to the superpotential proportional to $q_{1} q_{2}$ is

$$
\begin{aligned}
\left.w_{q_{1} q_{2}}\right|_{\eta_{Q_{1}}}= & -\sum_{s \in \mathcal{N}_{1}} \frac{(-1)^{n_{1}+n_{2}}}{\prod_{r \in \widehat{\mathcal{N}}_{1} \cup \mathcal{N}_{3}}\left(a_{s}-a_{r}\right)} \\
& \times \frac{1}{\prod_{t \in \mathcal{N}_{2}}\left(a_{s}-a_{t}\right)^{2}}
\end{aligned}
$$

$$
\begin{aligned}
& +\sum_{\substack{t_{1}, t_{2} \in \mathcal{N}_{2} \\
t_{1} \neq t_{2}}} \frac{(-1)^{n_{1}+n_{2}}}{\left(a_{t_{1}}-a_{t_{2}}\right) \prod_{r \in \mathcal{N}_{1} \cup \mathcal{N}_{3}}\left(a_{t_{1}}-a_{r}\right)} \\
& \times \frac{1}{\prod_{s \in \widehat{\mathcal{N}}_{2}}\left(a_{t_{1}}-a_{s}\right)^{2}} \\
& +\sum_{s \in \mathcal{N}_{1}} \sum_{t \in \mathcal{N}_{2}} \frac{(-1)^{n_{1}+n_{2}}}{\left(a_{t}-a_{s}\right) \prod_{r \in \mathcal{N}_{1} \cup \mathcal{N}_{3}}\left(a_{t}-a_{r}\right)} \\
& \times \frac{(-1)^{n_{1}+n_{2}}}{\prod_{s \in \widehat{\mathcal{N}}_{2}}\left(a_{t}-a_{s}\right)^{2}} \\
& +\sum_{\substack{t_{1}, t_{2} \in \mathcal{N}_{2} \\
t_{1} \neq t_{2}}} \frac{1}{\left(a_{t_{1}}-a_{t_{2}}\right) \prod_{r \in \widehat{\mathcal{N}}_{2} \cup \mathcal{N}_{3}}\left(a_{t_{1}}-a_{r}\right)} \\
& \times \frac{1}{\prod_{s \in \mathcal{N}_{1} \cup \widehat{\mathcal{N}}_{2}\left(a_{t_{2}}-a_{s}\right)} .}
\end{aligned}
$$

Similarly, for the quiver $Q_{2}$ we find:

$$
\begin{aligned}
& \left.w_{q_{1} q_{2}}\right|_{\eta_{Q_{2}}}=+\sum_{u \in \mathcal{N}_{3}} \frac{(-1)^{n_{1}+n_{2}}}{\prod_{r \in \mathcal{N}_{1} \cup \widehat{\mathcal{N}}_{3}}\left(a_{u}-a_{r}\right)} \\
& \times \frac{1}{\prod_{t \in \mathcal{N}_{2}}\left(a_{u}-a_{t}\right)^{2}} \\
& -\sum_{\substack{t_{1}, t_{2} \in \mathcal{N}_{2} \\
t_{1} \neq t_{2}}} \frac{(-1)^{n_{1}+n_{2}}}{\left(a_{t_{1}}-a_{t_{2}}\right) \prod_{r \in \mathcal{N}_{1} \cup \mathcal{N}_{3}}\left(a_{t_{1}}-a_{r}\right)} \\
& \times \frac{1}{\prod_{s \in \widehat{\mathcal{N}}_{2}}\left(a_{t_{1}}-a_{S}\right)^{2}} \\
& -\sum_{t \in \mathcal{N}_{2}} \sum_{u \in \mathcal{N}_{3}} \frac{(-1)^{n_{1}+n_{2}}}{\left(a_{t}-a_{u}\right) \prod_{r \in \mathcal{N}_{1} \cup \mathcal{N}_{3}}\left(a_{t}-a_{r}\right)} \\
& \times \frac{1}{\prod_{s \in \widehat{\mathcal{N}}_{2}}\left(a_{t}-a_{s}\right)^{2}} \\
& -\sum_{\substack{t_{1}, t_{2} \in \mathcal{N}_{2} \\
t_{1} \neq t_{2}}} \frac{(-1)^{n_{1}+n_{2}}}{\left(a_{t_{1}}-a_{t_{2}}\right) \prod_{r \in \mathcal{N}_{1} \cup \widehat{\mathcal{N}}_{2}}\left(a_{t_{1}}-a_{r}\right)} \\
& \times \frac{1}{\prod_{s \in \widehat{\mathcal{N}}_{2} \cup \mathcal{N}_{3}}\left(a_{t_{2}}-a_{s}\right)} .
\end{aligned}
$$

Once again, we have found perfect agreement, term by term, between these results and those obtained by solving the twisted chiral ring equations for the quivers $Q_{1}$ and $Q_{2}$.

\section{References}

1. S. Gukov, Surface Operators. arXiv: 1412.7127

2. S. Gukov, E. Witten, Gauge Theory, Ramification, and the Geometric Langlands Program. arXiv:hep-th/0612073

3. S. Gukov, E. Witten, Rigid surface operators. Adv. Theor. Math. Phys. 14(1), 87-178 (2010). arXiv:0804.1561

4. D. Gaiotto, Surface operators in $N=2$ dd Gauge theories. JHEP 11, 090 (2012). arXiv:0911.1316 
5. D. Gaiotto, S. Gukov, N. Seiberg, Surface defects and resolvents. JHEP 09, 070 (2013). arXiv:1307.2578

6. H. Kanno, Y. Tachikawa, Instanton counting with a surface operator and the chain-saw quiver. JHEP 06, 119 (2011). arXiv:1105.0357

7. A. Gorsky, B. Le Floch, A. Milekhin, N. Sopenko, Surface defects and instanton? Vortex interaction. Nucl. Phys. B 920, 122-156 (2017). arXiv: 1702.03330

8. S.K. Ashok, M. Billo, E. Dell'Aquila, M. Frau, R.R. John, A. Lerda, Modular and duality properties of surface operators in $N=2 *$ gauge theories. JHEP 07, 068 (2017). arXiv: 1702.02833

9. S.K. Ashok, M. Billo, E. Dell'Aquila, M. Frau, V. Gupta, R.R. John, A. Lerda, Surface operators in 5d gauge theories and duality relations. JHEP 05, 046 (2018). arXiv: 1712.06946

10. S.K. Ashok, M. Billo, E. Dell'Aquila, M. Frau, V. Gupta, R.R. John, A. Lerda, Surface operators, chiral rings and localization in $N=2$ gauge theories. JHEP 11, 137 (2017). arXiv:1707.08922

11. L.F. Alday, D. Gaiotto, S. Gukov, Y. Tachikawa, H. Verlinde, Loop and surface operators in $N=2$ gauge theory and Liouville modular geometry. JHEP 1001, 113 (2010). arXiv:0909.0945

12. L.F. Alday, Y. Tachikawa, Affine SL(2) conformal blocks from 4d gauge theories. Lett. Math. Phys. 94, 87-114 (2010). arXiv: 1005.4469

13. E. Witten, Phases of $N=2$ theories in two-dimensions. Nucl. Phys. B 403, 159-222 (1993). arXiv:hep-th/9301042

14. O. Aharony, A. Hanany, K.A. Intriligator, N. Seiberg, M.J. Strassler, Aspects of $N=2$ supersymmetric gauge theories in three-dimensions. Nucl. Phys. B 499, 67-99 (1997). arXiv:hep-th/9703110

15. A. Hanany, K. Hori, Branes and $N=2$ theories in two-dimensions. Nucl. Phys. B 513, 119-174 (1998). arXiv:hep-th/9707192

16. N. Nekrasov, BPS/CFT correspondence IV: sigma models and defects in gauge theory. arXiv:1711.11011

17. S. Jeong, N. Nekrasov, Opers, Surface Defects, and Yang-Yang Functional. arXiv: 1806.08270

18. N. Seiberg, Electric-magnetic duality in supersymmetric non Abelian gauge theories. Nucl. Phys. B 435, 129-146 (1995). arXiv:hep-th/9411149

19. F. Benini, D.S. Park, P. Zhao, Cluster Algebras from Dualities of $2 \mathrm{~d} N=(2,2)$ Quiver Gauge Theories. Commun. Math. Phys. 340, 47-104 (2015). arXiv:1406.2699
20. J.C. Jeffrey, F.C. Kirwan, Localization for non-abelian actions. Topology 34, 291-327 (1995). arXiv:alg-geom/9307001

21. N. Nekrasov, Seiberg-Witten prepotential from instanton counting. Adv. Theor. Math. Phys. 7, 831-864 (2004). arXiv:hep-th/0206161

22. N. Nekrasov, A. Okounkov, Seiberg-Witten theory and random partitions. Prog. Math. 244, 525-596 (2006). arXiv:hep-th/0306238

23. N.A. Nekrasov, S.L. Shatashvili, Quantum integrability and supersymmetric vacua. Prog. Theor. Phys. Suppl. 177, 105-119 (2009). arXiv:0901.4748

24. N. Nekrasov, S. Shatashvili, Quantization of Integrable Systems and Four Dimensional Gauge Theories. arXiv:0908.4052

25. U. Bruzzo, F. Fucito, J.F. Morales, A. Tanzini, Multi-instanton calculus and equivariant cohomology. JHEP 05, 054 (2003). arXiv:hep-th/0211108

26. A.S. Losev, A. Marshakov, N.A. Nekrasov, Small Instantons, Little Strings and Free Fermions. arXiv:hep-th/0302191

27. R. Flume, F. Fucito, J.F. Morales, R. Poghossian, Matone's relation in the presence of gravitational couplings. JHEP 04, 008 (2004). arXiv:hep-th/0403057

28. M. Billo, M. Frau, F. Fucito, L. Giacone, A. Lerda, J.F. Morales, D. Ricci-Pacifici, Non-perturbative gauge/gravity correspondence in $N=2$ theories. JHEP 1208, 166 (2012). arXiv: 1206.3914

29. S.K. Ashok, M. Billo, E. Dell'Aquila, M. Frau, A. Lerda, M. Moskovic, M. Raman, Chiral observables and S-duality in $N=$ $2 * \mathrm{U}(\mathrm{N})$ gauge theories. JHEP 11, 020 (2016). arXiv:1607.08327

30. D. Orlando, S. Reffert, Relating Gauge theories via Gauge/Bethe correspondence. JHEP 1010, 071 (2010). arXiv:1005.4445

31. F. Benini, R. Eager, K. Hori, Y. Tachikawa, Elliptic genera of twodimensional $N=2$ gauge theories with rank-one gauge groups. Lett. Math. Phys. 104, 465-493 (2014). arXiv:1305.0533

32. K. Hori, H. Kim, P. Yi, Witten index and wall crossing. JHEP 01, 124 (2015). arXiv:1407.2567

33. C. Hwang, P. Yi, Y. Yoshida, Fundamental vortices. Wallcrossing, and particle-vortex duality. JHEP 05, 099 (2017). arXiv:1703.00213 